\title{
THE PRODUCTION OF RESIDUAL NUCLEI IN PERIPHERAL HIGH ENERGY NUCLEUS-NUCLEUS INTERACTIONS
}

\author{
A. Ferrari, P.R. Sala \\ INFN, Sezione di Milano, Via Celoria 16, I-20133 Milano, Italy \\ J. Ranft \\ Departamento de Fisica de Particulas, Universidade de Santiago de Compostela, \\ E-15706 Santiago de Compostela, Spain \\ and \\ S. Roesler \\ Universität Siegen, Fachbereich Physik, D-57068 Siegen, Germany
}

\begin{abstract}
A formation zone intranuclear cascade model is applied to peripheral nucleus-nucleus collisions. We calculate the excitation energies of prefragments, treat their further nuclear disintegration and introduce a model for nuclear deexcitation by photon emission. Results are compared to data on target associated particle production in nucleus-nucleus collisions. We discuss implications of these models to the description of particle production in the fragmentation regions. Special emphasis is put on applications for air showers induced by cosmic ray nuclei and for residual nucleus production at heavy ion colliders.
\end{abstract}

Siegen SI 96-02

Santiago de Compostela US-FT/9-96

March 1996 


\section{Introduction}

High energy nucleus-nucleus collisions can be described by multiple scattering processes between nucleons of the interacting nuclei in which most of the final state particles are produced. These particles either escape directly from the interaction region or may induce intranuclear cascade processes, i.e. they may "knock out" further nucleons while penetrating through the spectator parts of the nuclei. Both, the primary and secondary nucleon-nucleon interactions and the intranuclear cascade contribute to the excitation of the spectator fragments. After peripheral collisions their deexcitation proceeds via evaporating nucleons and light fragments and via photon-emission until a stable configuration is reached, which we call "residual nucleus". In contrast, for low impact parameters or small, highly excited prefragments other nuclear disintegration processes, such as multifragmentation, become important.

Primary and secondary interactions between nucleons of both nuclei dominate the hadron production in most of the rapidity region covered by the interaction and are well described in the framework of the Dual Parton Model (DPM) [四]. They were extensively studied with the Monte Carlo (MC) implementations of this model DTUnuC [2] and DPMJET-II [3]. However, when dealing with particle or fragment production in the forward or backward fragmentation regions a detailed description of intranuclear cascade processes and of nuclear disintegration is of particular importance. Examples are air showers induced by high energy cosmic ray nuclei or aspects of cascade processes in matter initiated by heavy ions in general. A formation zone intranuclear cascade model, the calculation of nuclear excitation energies, and models for nuclear evaporation, high energy fission and the break-up of light nuclei were discussed in [4]. It was shown that their MC-realizations for hadron-nucleus interactions describe successfully the basic features of target associated particle production.

In this paper, these models are applied to nucleus-nucleus collisions and extended by a model for $\gamma$-deexcitation. We stress, that the formation zone intranuclear cascade model and the calculation of nuclear excitation energies as they are formulated and presented here can only be expected to work reliably in peripheral collisions. Nevertheless, we apply them in minimum bias situations to all collisions, since the fraction of central collisions, where they might fail is rather small. It should definitely not be applied to central collisions. Most of the data, we have to compare with are for asymmetric collisions of typically one light projectile nucleus with a heavier target nucleus. It seems, that the model performs rather well in such collisions. We do however not know, and there are so far no data to compare with, how well the model performs in collisions of identical or nearly identical heavy nuclei.

In Sect.2 we summarize briefly the main steps of sampling nucleus-nucleus interactions within the event generators DTUNUC 2.0 and DPMJET-II. Furthermore, we summarize the basic ideas of the formation zone intranuclear cascade model, describe the application to nucleus-nucleus collisions and calculate nuclear excitation energies. In Sect.3 a model for nuclear deexcitation by photon emission is introduced and its MC-implementation is discussed. In Sect. 4 the production of slow, target associated particles is compared to experimental data. Cross sections for the production of residual nuclei are calculated and compared to measured cross sections. In Sect.5 we discuss applications to air showers induced by cosmic ray nuclei and give cross sections for residual nuclei production in interactions with air. Furthermore, mass distributions of residual nuclei produced in lead-lead collisions at RHIC-energies are 
discussed. Finally, in Sect.6 we summarize our results.

\section{The formation zone intranuclear cascade model for nucleus-nucleus collisions}

\subsection{The event generators DPMJET-II and DTUNUC 2.0}

Both MC event generators, DPMJET-II and DTUNUC 2.0, start with sampling the spatial initial configuration, i.e. the positions of the nucleons in space-time in the rest system of the corresponding nucleus, from standard density distributions. The nucleons are assumed to behave like a Fermi-gas. Therefore, the momenta assigned to the nucleons are sampled from zero-temperature Fermi distributions.

The collision proceeds via $\nu$ nucleon-nucleon interactions between $\nu_{p}$ and $\nu_{t}$ nucleons from the projectile and target, resp. The values $\nu, \nu_{p}, \nu_{t}$, and the impact parameter of the nucleusnucleus collision are obtained according to the Glauber-theory using the MC-algorithm of [5]. Particle production in each nucleon-nucleon interaction is described by the DPM but based on different MC-realizations in the two event generators. The DPMJET-II code includes the DTUJET-93 [6] event generator for hadron-hadron interactions, whereas in the DTUNUC 2.0 code a one-Pomeron exchange model for low energies or the PHOJET [7, 8, 9] description of the DPM can be chosen optionally. The application of DPMJET-II and DTUNUC 2.0 is therefore restricted to upper energy limits of about $43 \mathrm{TeV}$ and $1 \mathrm{TeV}$ [ [8] c.m. energy per nucleon up to which the models DTUJET and PHOJET are valid. For details we refer to the literature.

Using a quasi-classical description, the hadrons produced in the $\nu$ nucleon-nucleon interactions are not only known with their 4-momentum but also with their position in space-time. They may cause intranuclear cascade processes in the spectator parts of the nuclei which were not involved in the primary and secondary interactions. Both mechanisms of particle production, the $\nu$ nucleon-nucleon interactions and the intranuclear cascade contribute to the excitation of the spectators. The parts of the code concerning the formation zone intranuclear cascade, the calculation of excitation energies and the various models treating the disintegration and deexcitation of prefragments, which are subject to this work, are identical in DPMJET-II and DTUNUC 2.0.

\subsection{The formation zone cascade in the spectator prefragments}

A description of how to implement the formation zone intranuclear cascade [11, [12] in hadronnucleus collisions was given in $[4$. Therefore, in this Section we summarize only the basic ideas.

Within the quark model, the states being formed in the primary nucleon-nucleon interaction can be understood as consisting of valence quarks only, i.e without the full system of sea quarks, antiquarks, and gluons and have therefore a reduced probability for hadronic reinteractions inside the nucleus [1]. These reduced interaction probabilities can be taken

\footnotetext{
${ }^{1}$ An extension to higher energies is planned for the near future [10]
} 
into account by assigning formation times to the hadrons produced[2]. The hadrons are not able to reinteract with nucleons of the residual spectator nuclei within that time. For each secondary a formation time $\tau$ in its rest system is sampled from an exponential distribution with an average value $\tau_{s}$ defined by [4, 12]

$$
\tau_{s}=\tau_{0} \frac{m_{s}^{2}}{m_{s}^{2}+p_{s \perp}^{2}} .
$$

$m_{s}$ and $p_{s \perp}$ are the mass and the transverse momentum of the secondary and $\tau_{0}$ is a free parameter which has to be adjusted by comparing particle production within the model to experimental data. We fix $\tau_{0}$ at a value of $1.9 \mathrm{fm} / c$. After having assigned a formation time to a secondary its spatial positions in the rest system of both nuclei are known and we start with considering an intranuclear cascade step in one (randomly chosen) of the spectators.

Due to relativistic time dilatation secondaries with high energies in the rest frame of the considered nucleus are mostly formed outside of the spectator part of this nucleus whereas those with low energies are formed inside. The latter may penetrate the spectator and initiate intranuclear cascade processes. We note that this scheme is not Lorentz invariant. However, the dependence of the results on the choice of the particular Lorentz frame is assumed to be small. Therefore we avoid a generally possible but technically cumbersome Lorentz invariant formulation of the intranuclear cascade within the present approach. Elastic and inelastic interactions with spectator nucleons are treated using the MC-model HADRIN [13]. This code is based on measured cross sections and interaction channels up to a laboratory momentum of $5 \mathrm{GeV}$. We apply HADRIN to hadron-nucleon interactions up to $9 \mathrm{GeV}$ and neglect those at higher energies. Reinteractions beyond $5 \mathrm{GeV}$ occur much less frequently than reinteractions below $5 \mathrm{GeV}$ and a more detailed treatment would not change the results discussed in this paper. Furthermore, the absorption of low-energy mesons and antiprotons by interactions with two-nucleon systems and Pauli's principle are taken into account [2]. If there was no interaction possible in the considered spectator we proceed with sampling a cascade step in the other spectator.

For secondaries produced in intranuclear cascade processes we apply the same formalism, i.e. a formation time is sampled, the secondary is transported to the end of the formation zone and reinteractions are treated if they are possible. Due to these intranuclear cascade processes nucleons are knocked out of the residual spectator nuclei if their energy is high enough to escape from the nuclear potential which will be discussed in the following section.

\subsection{The calculation of nuclear excitation energies}

Excitation energies of projectile and target prefragments in nucleus-nucleus collisions are calculated in a way which is similar to the one described in [4] for hadron-nucleus collisions.

We assume that nucleons of a nucleus of mass $A$ and charge $Z$ move in an effective nuclear

\footnotetext{
${ }^{2}$ In general one would have to consider cascade processes initiated by resonances instead of those initiated by stable hadrons. But since interaction cross sections of resonances are less well known we start from hadrons and compensate the effects of this approximation by using an effective formation time
} 
potential defined by a sum of a Fermi-potential and a binding energy contribution

$$
V_{i}(A, Z)=\frac{\left[p_{i}^{\mathrm{F}}(A, Z)\right]^{2}}{2 m_{i}}+E_{i}^{\mathrm{bind}}(A, Z), \quad i=\text { proton, neutron }
$$

with

$$
\begin{aligned}
p_{i}^{\mathrm{F}}(A, Z) & =\alpha_{\text {mod }}^{\mathrm{F}}\left[\frac{3 h^{3}}{8 \pi} \frac{N_{i}}{V_{\mathrm{A}}}\right]^{\frac{1}{3}} \\
E_{p}^{\text {bind }}(A, Z) & =E_{\text {bind }}(A, Z)-E_{\text {bind }}(A-1, Z-1) \\
E_{n}^{\text {bind }}(A, Z) & =E_{\text {bind }}(A, Z)-E_{\text {bind }}(A-1, Z)
\end{aligned}
$$

and $N_{p}=Z, N_{n}=A-Z$. $V_{\mathrm{A}}$ stands for the volume of the corresponding nucleus with an approximate nuclear radius $R_{A}=r_{0} A^{1 / 3}, r_{0}=1.29 \mathrm{fm}$, and $E_{\mathrm{bind}}$ for its binding energy. Modifications of the actual nucleon momentum distribution arising, for instance, from nuclear skin effects are taken into account by the reduction factor $\alpha_{\text {mod }}^{\mathrm{F}}$. This factor is adjusted by comparing the results of the model to measured black particle production (see Section 4.1) and is fixed at $0.55^{5}$. To be able to escape, positively charged particles have to penetrate a Coulomb-barrier given by

$$
V_{\mathrm{C}}(A, Z)=\frac{e^{2}}{4 \pi \epsilon_{0} r_{0}} \frac{Z}{\left(1+A^{1 / 3}\right)} .
$$

$e$ is the elementary charge and $r_{0}=1.29 \mathrm{fm}$.

Due to the primary and secondary nucleon-nucleon interactions and to the intranuclear cascade processes the colliding nuclei lose part of their nucleons. These nucleons and the secondary hadrons which were formed inside one of the nuclei have to escape from the effective nuclear potential. Within our simplified approach the precise nuclear potentials of the spectators during or after the primary or secondary nucleon-nucleon interactions cannot be

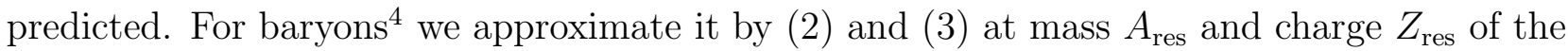
corresponding nuclear spectator (prefragment) replacing in (3) the modification factor $\alpha_{\bmod }^{\mathrm{F}}$ by $\tilde{\alpha}_{\text {mod }}^{\mathrm{F}}=\alpha_{\text {mod }}^{\mathrm{F}}+0.1$. In case of mesons we apply an effective potential of $0.002 \mathrm{GeV}$. The escape of a hadron from the nuclear potential of a prefragment implies that it transfers a recoil momentum to this prefragment. To be definite, this means, e.g. for a nucleon "leaving" the projectile prefragment, that the energy of this nucleon in the projectile rest system is reduced by the effective nuclear potential and, in case of a proton, has to be sufficiently high to overcome the Coulomb-barrier (田). Its 3-momentum is rescaled correspondingly. Energymomentum conservation requires a recoil momentum transferred to the prefragment which is equal to this correction. Adding up these recoil momenta separately for the projectile and target spectator, $\left(E_{\mathrm{rcl}}, \vec{p}_{\mathrm{rcl}}\right)$, one obtains the 4-momenta of the prefragments

$$
\left(E_{\mathrm{res}}, \vec{p}_{\mathrm{res}}\right)=\left(M_{\mathrm{A}}, \overrightarrow{0}\right)-\sum_{i=1}^{N_{w}}\left(E_{i}^{\mathrm{F}}, \vec{p}_{i}^{\mathrm{F}}\right)+\left(E_{\mathrm{rcl}}, \vec{p}_{\mathrm{rcl}}\right) .
$$

\footnotetext{
${ }^{3} \mathrm{In}$ 泪 we used $\alpha_{\text {mod }}^{\mathrm{F}}=0.75$ together with $\tau_{0}=2 \mathrm{fm} / c$, which were obtained comparing the model to h-A data only. The parameters reported in this paper are based on a larger data set, i.e. are based on both, data from hadron-nucleus and nucleus-nucleus collisions

${ }^{4}$ The fraction of baryons other than nucleons which are created inside of one of the nuclei is small. They are assumed to move in a nucleon potential
} 
$N_{w}$ is the number of wounded nucleons in the corresponding nucleus and $M_{\mathrm{A}}$ is the mass of this nucleus. For simplicity we omit indices referring the quantities to projectile and target.

The excitation energy $U$ of a prefragment is defined as the energy above the ground state mass $E_{0}$

$$
\begin{array}{cl}
U & =E_{\text {res }}-E_{0}\left(A_{\text {res }}, Z_{\text {res }}\right) \\
E_{0}\left(A_{\text {res }}, Z_{\text {res }}\right) & =Z_{\text {res }} m_{\mathrm{p}}+\left(A_{\text {res }}-Z_{\text {res }}\right) m_{\mathrm{n}}-E_{\text {bind }}\left(A_{\text {res }}, Z_{\text {res }}\right) .
\end{array}
$$

with $E_{\text {bind }}$ being the corresponding binding energy which is either the experimentally determined excess mass or is obtained from mass formulae for nuclides far from the stable region. As examples, in Table 1 we give average excitation energies and excitation energies per nucleon of target prefragments after interactions of oxygen and aluminum with gold nuclei at different energies. In asymmetric nucleus-nucleus interactions the excitation energies of the prefragments of the heavier target nucleus are slowly increasing with the beam energy and, at fixed energy, with the mass number of the projectile.

\section{$3 \gamma$-deexcitation of residual spectator nuclei}

At the end of the intranuclear cascade the prefragments are supposed to be left in an equilibrium state, characterized by their masses, charges, and excitation energies with no further memory of the steps which led to their formation. Since the excitation energies can be higher than the separation energies, nucleons and light fragments $\left(\alpha, \mathrm{d},{ }^{3} \mathrm{H},{ }^{3} \mathrm{He}\right)$ can still be emitted. A detailed account on the evaporation treatment which follows the intranuclear cascade was given in [4]. Furthermore, a model for high energy fission and a Fermi Break-up model for light nuclei where an excited prefragment is supposed to disassemble just in one step into two or more fragments were discussed in [4].

The evaporation stage ends when the nuclear excitation energy becomes lower than all separation energies for nucleons and fragments. This residual excitation energy is then dissipated through emission of photons. In reality, photon emission occurs even during the preequilibrium and evaporation stages, in competition with particle emission, but its relative probability is low, and it is presently neglected in the model.

$\gamma$-deexcitation proceeds through a cascade of consecutive photon emissions, until the ground state is reached. The cascade is assumed to be statistical as long as the excitation energy is high enough to allow the definition of a continuous nuclear level density. Below a (somewhat arbitrary) threshold, set at the pairing gap value, the cascade goes through transitions between discrete levels.

The statistical model formulation for the $\gamma$-ray emission probability is again similar to those for evaporation and fission [14, 15

$$
P\left(E_{\gamma}\right) d E_{\gamma}=\frac{\rho_{f}\left(U_{f}\right)}{\rho_{i}\left(U_{i}\right)} \sum_{L} f\left(E_{\gamma}, L\right) d E \gamma
$$

where $L$ is the multipolarity of the $\gamma$ transition. The strength functions $f(E, L)$ can be either derived from photoabsorption cross sections or calculated from single-particle estimates of 
transition strengths. The former approach is more sophisticated but requires the knowledge of the resonance parameters for all isotopes; the latter approach is easier and sufficient for a first order estimate of the $\gamma$ spectral distribution. We thus assume

$$
f\left(E_{\gamma}, L\right)=c_{L} \cdot F_{L}(A) \cdot E_{\gamma}^{(2 L+1)}
$$

where $E_{\gamma}^{(2 L+1)}$ is the energy dependence for multipolarity $L$. For the $c_{L}$ coefficients we adopted the Weisskopf single particle estimates [16]. The $F_{L}(A)$ factors have been included to partially account for the many effects that bring to deviations from the single particle estimates. They are rough A dependent averages of the hindrance and enhancement factors given in [17]. Only E1, M1, and E2 transitions have been considered. The assumed level density $\rho$ is the same as in the evaporation part described in [4], but the ratio of exponentials coming from the level densities has been approximated as first order expansion around $E_{\gamma}=0$. This is equivalent to the assumption of a constant nuclear temperature at low excitation energies, which is often used in the analysis of photon emission following neutron capture [14, 18].

As a result, one obtains the expressions for the emission probabilities for the considered multipoles. Since competition between photon and particle emission is neglected at the present status of the model, only the relative values are of interest

$$
\begin{aligned}
P\left(L, E_{\gamma}\right) d E_{\gamma} & =\tilde{C}_{L} E_{\gamma}^{(2 L+1)} e^{\frac{E_{\gamma}}{T}} d E_{\gamma} \\
\frac{\tilde{C}_{M 1}}{\tilde{C}_{E 1}} & =0.31 A^{-\frac{2}{3}} \frac{F_{M 1}(A)}{F_{E 1}(A)} \\
\frac{\tilde{C}_{E 2}}{\tilde{C}_{E 1}} & =7.2 \cdot 10^{-7} A^{\frac{2}{3}} \frac{F_{E 2}(A)}{F_{E 1}(A)} \mathrm{MeV}^{-2}
\end{aligned}
$$

$T$ is the nuclear temperature at the initial excitation energy $U$, taken as $U-\Delta=a T^{2}$, $a$ being the usual level density constant (see Eq.15 of [4]) and $\Delta$ the pairing energy.

A first sampling is performed on the integrated photon emission probabilities to choose the character( electric or magnetic) and the multipole order of the emitted photon, and a second sampling is performed to determine the emission energy according to the selected multipolarity. For both steps the full energy range $0 \leq E_{\gamma} \leq U$ is used, even though the intrinsic limit of validity would be $0 \leq E_{\gamma} \leq(U-\Delta)$. After emission, all parameters are updated on the basis of the new excitation energy, and another statistical emission is performed, until the excitation energy falls below the preset "discrete level threshold". This threshold has been set to the pairing energy for even-even or odd mass nuclei; for odd-odd nuclei the threshold corresponds to the (known or approximated) first excited level.

For many isotopes the experimentally determined values of the first and second excited levels have been tabulated in the code, for the others a rotational-like structure is assumed, with level energy given by :

$$
U_{I}=\frac{\hbar^{2}}{2 \mathcal{I}} I(I+1)
$$

where $I$ is the level spin (integer for even-mass, half integer for odd mass nuclei, 0 or $1 / 2$ for the ground state), and $\mathcal{I}$ is the nuclear moment of inertia, taken as 0.4 times that of a rigid body . The last steps of the $\gamma$ cascade consist of $\delta I=2$ transitions among these rotational 
levels, down to the ground state. When known levels are tabulated, the cascade is forced to pass through them. All photons are emitted isotropically, since from the evaporation stage we have no information on the residual nucleus spin and polarization.

The $\gamma$ deexcitation model has been developed and tested within the cascade-preequilibriumevaporation model PEANUT [19, 20], and it has been easily coupled to DTUNUC and DPMJET since its algorithm depends only on the residual nucleus mass, $\mathrm{Z}$ and excitation energy after evaporation, not on the details of the preceding interaction history. Two examples of its performances are shown in Figs. 1 and 2. Both, the total photon multiplicity and the shape of the spectra are well reproduced.

\section{Comparing target-associated particle production to experimental data}

\subsection{Grey and black particles and correlations}

As discussed in Sect.2.2 nucleons might be knocked out by intranuclear cascade processes if their energies are sufficiently high to escape from the nuclear potential. The kinetic energies are typically of the order of 20 to $400 \mathrm{MeV}$ in the rest frame of the considered nucleus. In contrast, nucleons and light fragments evaporated from the excited prefragment possess less energy. This allows to study both mechanisms of low energy particle production rather independently, apart from the fact that in our model the cascade particles contribute to the excitation of the prefragment and, therefore, indirectly to the multiplicity of the evaporated particles.

There are only a few experiments known to us in which target associated particle production in interactions with nuclear projectiles is investigated. The most detailed results were obtained in experiments using emulsion targets. The emulsions usually consist of a component of light nuclei $(\mathrm{H}, \mathrm{C}, \mathrm{N}, \mathrm{O})$ and a component of heavy nuclei $(\mathrm{Ag}, \mathrm{Br})$. The appearance of slow charged particles and light fragments in these experiments has led to their subdivision into "grey" and "black" particles. Grey and black particles roughly coincide in energy with the above mentioned cascade and evaporation particles.

In Table 2 we give average values for multiplicities of grey and black particles in interactions of oxygen, silicon, sulfur, and gold nuclei with emulsions at different beam energies as calculated within our model. The experimental values are results of the EMU01-Collab. [21, 22]. Our results are superpositions of multiplicities obtained with hydrogen, light and heavy nuclear targets weighted with the fractions $0.13,0.31$, and 0.56 resp., corresponding to the emulsion composition used in the experiments [23]. Furthermore, we apply the definitions for "grey" and "black" as given by the EMU01-Collab. Grey particles are protons, pions, and kaons with kinetic energies between 26 and $375 \mathrm{MeV}, 12$ and $56 \mathrm{MeV}$, and 20 and $198 \mathrm{MeV}$, resp. Black particles are singly and multiply charged having lower energies than the grey particles. Apart from the results for $\left\langle N_{g}\right\rangle$ at low energies where the model seems to overestimate the measured multiplicities the agreement is satisfactory. Slow protons contribute about $79 \%$ and slow charged pions about $19 \%$ to the calculated grey particle multiplicity in S-Emulsion interactions at $200 \mathrm{GeV} /$ nucleon. 
In Figs.3 6 we show multiplicity distributions for grey and black particles in oxygenand sulfur-emulsion interactions at $200 \mathrm{GeV} /$ nucleon and in gold-emulsion interactions at 11.6 GeV/nucleon. They are compared to data of the EMU01-Collab. [22, 24] and, for oxygen and sulfur projectiles, in addition to data of the KLM-Collab. [25]. The contribution of the light emulsion component dominates the shapes of the distributions at low multiplicities, whereas for $\left\langle N_{g, b}\right\rangle \gtrsim 5$ the distributions are determined by interactions with the heavy target nuclei, silver and bromine. Again, within our calculations we have applied the above given definitions for "grey" and "black". The differences between the experimental data of both Collaborations can be possibly explained by the cut of 0.25 between the Lorentz- $\beta$ values of grey and black particles used by the KLM-Collab. 25]. For the oxygen- and sulfur-emulsion interactions (Figs. 3,5) our results agree with the measured distributions apart from a possible underestimation of the tail of the $N_{g}$-distributions at high multiplicities. In case of gold projectiles the calculated grey particle distribution (Fig. (1) shows for $N_{g}>10$ a rather flat shape, which is different from the measured distribution. This indicates more cascading and probably bigger target prefragments than seen in the experiment. This underlines the fact that our model of intranuclear cascading and excitation energy calculation is too simplified to understand slow particle production in very asymmetric nucleus-nucleus collisions in the rest system of the smaller nucleus. This nucleus gets almost completely disintegrated already in the primary and secondary interactions and a multifragmentation model could be more appropriate for the description of the nuclear disintegration processes.

The reasonable description of the multiplicity distributions in oxygen- and sulfur-emulsion interactions suggests that the model should be able to reproduce measured correlations between grey, black, and shower particle multiplicities. In Figs.7a,b we show the average number of grey and black particles depending on the number of shower particles. The agreement with the data [24] is reasonable except for the average number of black particles around $N_{s} \approx 200$. The correlations between heavy (=grey+black) and shower particle multiplicities are compared to data [24] in Figs.77c,d. Our model is able to reproduce the measured correlations within their statistical uncertainties. Finally, in Figs.8a,b we give the correlations between grey and black particle multiplicities. We obtain slightly less grey particles in high-multiplicity $\mathrm{S}-\mathrm{Ag} / \mathrm{Br}$ interactions than seen in the experiment.

In order to investigate the kinematics for slow particle production angular distributions of grey and black particles are compared to data on oxygen- (Figs.9a,c) and sulfur-emulsion interactions (Figs.9b,d) at $200 \mathrm{GeV} / \mathrm{c} /$ nucleon [21, 25]. Whereas in the forward direction protons dominate the angular distributions of grey particles, in the backward direction pions produced in intranuclear cascade processes become important. Parametrizing the angular distributions of grey particles with $f\left(\cos \Theta_{g}\right)=K \exp \left(b \cos \Theta_{g}\right)$ we obtain $K=0.39$ and $b=1.25$. The experimental values are $K=0.44$ and $b=0.92$, i.e. our results show a bigger slope. The angular distributions of black particles are in good agreement with the data which supports the fact that we correctly describe the recoil momentum transferred to the residual nucleus.

For a more detailed investigation of slow particle production it is needed to compare the MC-results to data obtained in experiments which (i) are using one kind of target nucleus rather than a mixture as the emulsions are and (ii) which are able to identify particles and may therefore separate slow protons and light fragments from pions. Such an experiment with 
oxygen projectile nuclei and several target nuclei was performed by the WA80-Collab. and results were published in [26]. These data are subject to the limited detector acceptance [26, 27, 28] and any comparisons of model-results which were not filtered through the acceptance functions have to be taken with care. The following cuts have been applied to our results in order to be as close to the experimental conditions as possible [26]: (i) an event is considered if the energy within a cone of $0.3^{\circ}$ is less than $88 \%$ of the beam energy and (ii) only protons and singly charged fragments having zenit angles between $60^{\circ}$ and $160^{\circ}$ and kinetic energies between $30 \mathrm{MeV} /$ nucleon and $400 \mathrm{MeV} /$ nucleon were taken into consideration. In Fig.10 we show the multiplicity distributions of slow fragments from oxygen-nucleus interactions at $200 \mathrm{GeV} / c /$ nucleon together with the WA80-data. Our results are normalized to the data.

\subsection{Residual target nuclei}

The heavy prefragments which are left after the evaporation of nucleons and light nuclei can be considered as heavy fragments produced in a spallation or deep spallation process. The high energy fission and Fermi Break-up models contribute to a further modification of the mass yield if these processes are physically possible. Since fragmentation of prefragments with masses above $A_{\text {res }}=18$ is not treated we cannot expect to agree with measured mass yields in nucleus-nucleus collisions. However, as it is shown in Fig.11 for carbon-silver interactions at $25.2 \mathrm{GeV} /$ nucleon our cross sections for the production of residual target nuclei differ by not more than a factor of two from the measured values [29] within the mass range between 30 and 100 . The rising yields of residual nuclei close to the target mass $\left(A_{T}-5 \leq A_{\text {res }} \leq A_{\mathrm{T}}\right)$ are not described within our model since we do not consider such processes like quasi-elastic scattering and treat the nuclear potential only in a rough manner (c.f. discussion of target fragmentation in proton-nucleus interactions in 四). The calculated yields at $A_{\text {res }}=2,3,4$ represent nuclei evaporated from the prefragments.

\section{Applications}

\subsection{Residual nuclei and high-energy photons from interactions of cosmic-ray nuclei in the atmosphere}

For studying cosmic ray cascades the forward region is of main importance. In case of nucleusair interactions this involves the projectile associated particle production and the fragmentation and deexcitation of the projectile prefragment. Since oxygen and iron nuclei are typical cosmic ray nuclei we restrict the following study to oxygen- and iron-air interactions. Of course, the interactions of all cosmic ray nuclei will be treated by our event generators, if they are used in a cosmic ray cascade code.

In order to investigate to which extent the results discussed in this paper depend on the MC-realization of the DPM we compare multiplicities obtained with DPMJET-II and DTUNUC 2.0. In Tables 3 and $⿴ 囗 十$ we present shower, grey, and black particle multiplicities (the definitions are the same as for the comparisons with the emulsion data in Section 4.1) in iron- and oxygen-air interactions at different laboratory momenta of the projectile. Fur- 
thermore, the average multiplicities of evaporation products and deexcitation photons from the projectile and target fragments are given. Since DTUNUC 2.0 cannot yet be applied to interactions at nucleon-nucleon c.m. energies beyond $1 \mathrm{TeV}\left(p_{\text {Lab }} \approx 533 \mathrm{TeV} / c\right)$ multiplicities obtained with this event generator are presented only for the two lower momenta, $0.2 \mathrm{~A} \mathrm{TeV} / c$ and $20 \mathrm{~A} \mathrm{TeV} / c$ (second lines). It can be concluded that, apart from the shower particle multiplicities, differences in the given multiplicities caused by the particular MC-realization of the DPM are smaller than the statistical uncertainties of the calculations.

The mass yields of residual projectile nuclei in oxygen-air interactions are shown for two different energies in Fig.12a. We observe that the yield of nuclei is shifted towards smaller mass numbers as the oxygen energy increases. Light nuclei $\left(A_{\text {res }} \leq 4\right)$ clearly dominate the residual nuclei with higher masses. Note, that the prefragments obtained after the intranuclear cascade were subject to both, the fragmentation by the evaporation and the Fermi Break-up models. Structures apparent in the mass yields are not due to statistical fluctuations but result from the fragmentation process. In Fig.12b be present the mass yields of residual projectile nuclei in iron-air interactions. We obtain higher yields of small nuclei with increasing energy whereas the shapes of the yields at the two energies are similar above $A_{\text {res }}=25$. We stress again that for $A_{\text {res }}>16$ apart from evaporation of light fragments no further fragmentation is treated. The yields close to the iron mass might be underestimated as it is discussed in Sect.4.2.

Within our MC-event generators there are two main mechanisms of photon production, the decay processes of hadrons and the nuclear deexcitation of the prefragments. In order to investigate to which extent they contribute to the total photon spectrum we show in Figs.13a,b the pseudorapidity distributions of photons from $\pi^{0}$-decay and from deexcitation processes separately. It is obvious that photon production by nuclear deexcitation contributes only a minor fraction to the total photon yield in forward and backward direction. Integrating the two peaks in the distributions of the deexcitation photons separately one obtains the average multiplicity of photons from the deexcitation of the projectile and target prefragments, which is directly correlated to their average excitation energies after the evaporation step. It demonstrates, that the average residual excitation energy remains about constant with the collision energy above $200 \mathrm{GeV} /$ nucleon and that the average residual excitation energy of the prefragments originating from the iron nuclei is significantly higher as compared to the one from air nuclei.

Finally, in Figs.14a,b we show the Feynman- $x_{F}$ distributions of photons in the nucleonnucleon c.m. frame at a laboratory energy of $200000 \mathrm{TeV} /$ nucleon. Again, the contribution of deexcitation photons can be neglected in comparison to the decay photons.

\subsection{Residual nuclei production at heavy-ion colliders}

As for cosmic ray cascades a realistic description of projectile and target associated particle production and of the production of residual nuclei becomes important if one is investigating particle production in the forward or backward fragmentation region. Furthermore, in detector simulations for heavy ion experiments, such as simulations of the performances of trigger Zero Degree Calorimeters, as well as in radiation shielding calculations for existing or planned heavy ion colliders a model for the production of residual nuclei is an essential ingredient for 
the estimation of particle cascades in matter initiated by nuclei.

In Fig.15 we give cross sections for residual nuclei produced in lead-lead collisions at a RHIC- c.m. energy of $200 \mathrm{GeV} /$ nucleon. The nuclei with masses up to $A_{\text {res }}=4$ are evaporation products. Apart from Fermi Break-up for the few light fragments no further fragmentation was performed, i.e. the cross sections shown are production cross sections for residual nuclei from one of the lead nuclei after the evaporation step.

Again, as it was shown in Sect.4.1 the models describe slow particle production in asymmetric collisions rather satisfactorily but it has still to be proven that they can equally well be applied to collisions of identical or nearly identical heavy nuclei.

\section{Summary and conclusions}

In the present paper, models for formation zone intranuclear cascades in spectator prefragments, the evaporation of nucleons and light fragments, high energy fission processes, and the fragmentation of light nuclei were discussed and applied to peripheral nucleus-nucleus collisions. Furthermore, we have introduced a model for nuclear deexcitation by photon emission.

Comparing our results to data on slow particle and fragment production it is shown that together with the two-component Dual Parton Model for nucleus-nucleus collisions main features of projectile and target associated particle production are well described. In particular, results of calculations with DPMJET-II and DTUNUC 2.0 were found to be in agreement with multiplicity and angular distributions of grey and black prongs and with correlations between grey, black, and shower particle multiplicities. Although we stress, that the model is only valid for peripheral collisions, we use it actually for minimum bias nucleus-nucleus collisions, where a small fraction of central collisions contributes, for which the model cannot be expected to be reliable. Therefore, the model results might deviate from data on multiplicity distributions of grey and black prongs and slow fragments at the high multiplicity tails and we might expect that the model misses some part of the residual nucleus and nuclear fragment distribution.

The $\gamma$-deexcitation model is compared to low energy data. It is discussed that with rising collision energy, the contribution from deexcitation photons becomes relatively unimportant as compared to photons from hadronic decays.

However, even in the absence of a multifragmentation model the production of residual nuclei turned out to be an acceptable approximation for fragment production within a MC-event generator to be used to describe hadronic interactions in cascade codes like FLUKA [20, 30, 31] or HEMAS [32]. Using the discussed models within the simulation of cosmic ray cascades allows to go beyond the "superposition model" [33 and similar approximations for the collisions of cosmic ray nuclei in the atmosphere. Likewise, in heavy ion experiments, the nuclear fragments and residual nuclei are often very important to simulate the performance of components of the detectors in extreme forward or backward direction, like the Zero Degree Calorimeters of some existing experiments. 


\section{Acknowledgements}

One of the authors (S.R.) is grateful to F.W. Bopp and R. Engel for stimulating discussions. S.R. acknowledges the collaboration with R. Engel on the link between the DTUNUC and PHOJET event generators. We thank H. Gutbrod, K.-H. Kampert, and E. Stenlund from the WA80-Collaboration for helpful comments on the EMU01- and WA80-data. 


\section{References}

[1] A. Capella, U. Sukhatme, C. I. Tan and J. Tran Thanh Van: Phys. Rep. 236 (1994) 227

[2] H.-J. Möhring and J. Ranft: Z. Phys. C52 (1991) 643

[3] J. Ranft: Phys. Rev. D51 (1995) 64

[4] A. Ferrari, J. Ranft, S. Roesler and P. R. Sala: Cascade particles, nuclear evaporation, and residual nuclei in high energy hadron-nucleus interactions, ENSLAPP-A-551/95, (nucl-th/9509039), accepted for publication in Z. Phys. C, 1996

[5] S. Y. Shmakov, V. V. Uzhinskii and A. M. Zadoroshny: Comp. Phys. Commun. 54 (1989) 125

[6] P. Aurenche, F. W. Bopp, R. Engel, D. Pertermann, J. Ranft and S. Roesler: Comp. Phys. Commun. 83 (1994) 107

[7] R. Engel: Z. Phys. C66 (1995) 203

[8] R. Engel and J. Ranft: Hadronic photon-photon collisions at high energies, ENSLAPPA-540/95 (hep-ph/9509373), submitted for publication in Phys. Rev. D, 1995

[9] R. Engel: Multiparticle Photoproduction within the two-component Dual Parton Model, in preparation, 1996

[10] R. Engel: private communication,

[11] J. Ranft: Phys. Rev. D37 (1988) 1842

[12] J. Ranft: Z. Phys. C43 (1989) 439

[13] K. Hänssgen and J. Ranft: Comp. Phys. Commun. 39 (1986) 37

[14] G. A. Bartholomew et al.: Advances in Nuclear Physics 7 (1975) 229

[15] I. Bergqvist and N. Starfelt: Progress in Nuclear Physics 11 (1970) 1

[16] D. H. Wilkinson: in Nuclear Spectroscopy B, ed. by F. Ajzenberg-Selove, Academic Press, New York, 1960

[17] P. M. Endt: Atomic Data and Nuclear Data Tables 26 (1981) 46

[18] W. Dilg, W. Schantl and H. Vonach: Nucl. Phys. A217 (1973) 269

[19] P. R. Sala and A. Ferrari: in Proceedings of the International Conference on Monte Carlo Simulation in High Energy and Nuclear Physics, MC'93, Tallahassee, U.S.A., 1993, ed. by P. Dragovitsch, S. L. Linn and M. Burbank, World Scientific, p. 277, 1994 
[20] A. Fassò, A. Ferrari, J. Ranft and P. R. Sala: FLUKA: Performances and Applications in the Intermediate Energy Range, in Proceedings of the Specialists Meeting on Shielding Aspects of Accelerators, Targets \& Irradiation Facilities, Arlington, U.S.A., 1994, published by OECD/NEA, p. 287, 1994

[21] EMU01 Collab.: M. I. Adamovich et al.: Phys. Lett. B262 (1991) 369

[22] EMU01 Collab.: M. I. Adamovich et al.: Phys. Lett. B363 (1995) 230

[23] EMU01 Collab.: M. I. Adamovich et al.: Phys. Lett. B201 (1988) 397

[24] EMU01 Collab.: M. I. Adamovich et al.: Z. Phys. C65 (1995) 421

[25] KLM Collab.: A. Dabrowska et al.: Phys. Rev. D47 (1993) 1751

[26] WA80 Collab.: R. Albrecht et al.: Phys. Lett. B307 (1993) 269

[27] A. Baden et al.: Nucl. Instrum. Methods 203 (1982) 189

[28] H. H. Gutbrod: private communication,

[29] N. T. Porile, G. T. Cole and C. Rudy: Phys. Rev. C19 (1979) 2288

[30] A. Fassò, A. Ferrari, J. Ranft and P. R. Sala: FLUKA: present status and future developments, in Proceedings of the IVth International Conference on Calorimetry in High Energy Physics, La Biodola (Elba), Italy, 1993, ed. by A. Menzione and A. Scribano, World Scientific, p. 493, 1994

[31] P. A. Aarnio et al.: in Proceedings of the International Conference on Monte Carlo Simulation in High Energy and Nuclear Physics, MC'93, Tallahassee, U.S.A., 1993, ed. by P. Dragovitsch, S. L. Linn and M. Burbank, World Scientific, p. 88, 1994

[32] G. Battistoni, C. Forti and J. Ranft: Astroparticle Phys. 3 (1995) 157

[33] C. Forti et al.: Phys. Rev. D42 (1990) 3668

[34] G. L. Morgan: Report ORNL-5563, 1979

[35] J. K. Dickens, T. A. Love and G. L. Morgan: Report ORNL-4847, 1973

[36] EMU01 Collab.: M. I. Adamovich et al.: Phys. Lett. B230 (1989) 175 


\section{Tables}

Table 1: Average excitation energies and excitation energies per nucleon of target prefragments produced in oxygen- and aluminum-gold interactions before evaporation are given for different momenta per nucleon of the projectile nucleus.

\begin{tabular}{|c||c|c||c|c|}
\hline \multicolumn{1}{|c||}{} & \multicolumn{2}{c||}{ O-Au } & \multicolumn{2}{c|}{$\mathrm{Al}-\mathrm{Au}$} \\
$p_{\text {Lab }}$ & $\langle U\rangle$ & $\left\langle\frac{U}{A \text { res }}\right\rangle$ & $\langle U\rangle$ & $\left\langle\frac{U}{A \text { res }}\right\rangle$ \\
$(\mathrm{A} \mathrm{GeV} / c)$ & $(\mathrm{MeV})$ & $(\mathrm{MeV})$ & $(\mathrm{MeV})$ & $(\mathrm{MeV})$ \\
\hline \hline 20 & 592.0 & 5.6 & 550.0 & 5.9 \\
\hline 30 & 554.0 & 5.5 & 537.0 & 6.0 \\
\hline 50 & 567.0 & 5.6 & 546.0 & 6.2 \\
\hline 100 & 567.0 & 5.7 & 573.0 & 6.7 \\
\hline 200 & 573.0 & 5.8 & 591.0 & 7.1 \\
\hline 500 & 603.0 & 6.4 & 606.0 & 7.1 \\
\hline 1000 & 619.0 & 6.6 & 621.0 & 7.3 \\
\hline 2000 & 653.0 & 6.7 & 652.0 & 7.7 \\
\hline
\end{tabular}


Table 2: Multiplicities of grey $\left(N_{g}\right)$ and black $\left(N_{b}\right)$ particles in interactions of nuclei with emulsions are given for different projectile nuclei and for different projectile energies. The experimental data from the EMU01-Collab. were taken from [21] if not explicitly indicated in the table.

\begin{tabular}{|c|c||c|c|c|c|}
\hline & \multicolumn{2}{|c||}{$E_{\text {Lab }}$} & \multicolumn{2}{c|}{$\left\langle N_{g}\right\rangle$} & \multicolumn{2}{c|}{$\left\langle N_{b}\right\rangle$} \\
& $(\mathrm{GeV} /$ nucleon $)$ & DTUNUC & Exp. & DTUNUC & Exp. \\
\hline \hline O-Emul. & 14.6 & 6.1 & $5.2 \pm 0.2$ & 4.7 & $4.8 \pm 0.2$ \\
\hline & 60 & 4.3 & $5.7 \pm 0.4$ & 4.4 & $4.5 \pm 0.2$ \\
\hline & 200 & 4.2 & $4.3 \pm 0.3$ & 4.3 & $4.1 \pm 0.2$ \\
\hline Si-Emul. & 14.6 & 7.4 & $5.4 \pm 0.3$ & 4.6 & $4.6 \pm 0.2$ \\
\hline S-Emul. & 200 & 4.4 & $4.7 \pm 0.3$ & 4.2 & $3.9 \pm 0.2$ \\
\hline Au-Emul. & 11.6 & 9.7 & $5.8 \pm 0.19$ [22] & 3.6 & $3.52 \pm 0.12$ [22] \\
\hline
\end{tabular}

Table 3: Multiplicities of shower $\left(N_{s}\right)$, grey $\left(N_{g}\right)$, and black $\left(N_{b}\right)$ particles in iron-air interactions are given for different momenta per nucleon in the laboratory frame. In addition, multiplicities of evaporated protons $\left(N_{p}\right)$, neutrons $\left(N_{n}\right)$, and heavy fragments $\left(N_{h f}\right)$ and deexcitation photons $\left(N_{\gamma}\right)$ are presented for the projectile (iron) and target (air $=\mathrm{N}$ ) fragments. At $0.2 \mathrm{~A} \mathrm{TeV} / c$ and $20 \mathrm{~A} \mathrm{TeV} / c$ we give in the first line the multiplicities calculated with DPMJET-II and in the second line those calculated with DTUnUC 2.0. At the two highest energies only DPMJET-II predictions are given.

\begin{tabular}{|c|c|c|c|c|c|c|c|c|c|c|c|}
\hline \multirow{2}{*}{$\begin{array}{c}p_{\text {Lab }} \\
(\mathrm{A} \mathrm{TeV} / c) \\
\end{array}$} & \multirow[b]{2}{*}{$\left\langle N_{s}\right\rangle$} & \multirow[b]{2}{*}{$\left\langle N_{g}\right\rangle$} & \multirow[b]{2}{*}{$\left\langle N_{b}\right\rangle$} & \multicolumn{4}{|c|}{ Projectile fragments } & \multicolumn{4}{|c|}{ Target fragments } \\
\hline & & & & $\left\langle N_{p}\right\rangle$ & $\left\langle N_{n}\right\rangle$ & $\left\langle N_{\gamma}\right\rangle$ & $\left\langle N_{h f}\right\rangle$ & $\left\langle N_{p}\right\rangle$ & $\left\langle N_{n}\right\rangle$ & $\left\langle N_{\gamma}\right\rangle$ & $\left\langle N_{h f}\right\rangle$ \\
\hline 0.2 & 82.8 & 1.3 & 1.7 & 2.5 & 4.0 & 1.9 & 2.5 & 0.54 & 0.62 & 0.10 & 1.2 \\
\hline & 78.1 & 1.4 & 1.7 & 2.2 & 4.1 & 2.0 & 2.4 & 0.51 & 0.58 & 0.10 & 1.1 \\
\hline 20 & 256.5 & 1.2 & 1.6 & 2.8 & 4.5 & 1.7 & 2.9 & 0.57 & 0.65 & 0.08 & 1.2 \\
\hline & 234.6 & 1.4 & 1.6 & 2.7 & 4.6 & 1.8 & 2.8 & 0.55 & 0.63 & 0.08 & 1.1 \\
\hline 2000 & 641.3 & 1.1 & 1.5 & 3.2 & 4.9 & 1.4 & 3.2 & 0.56 & 0.65 & 0.07 & 1.1 \\
\hline 200000 & 1260.9 & 1.1 & 1.5 & 3.5 & 5.2 & 1.2 & 3.4 & 0.58 & 0.65 & 0.07 & 1.1 \\
\hline
\end{tabular}


Table 4: As in caption of Table 3 but for oxygen-air interactions.

\begin{tabular}{|c||c|c|c||c|c|c|c||c|c|c|c|}
\hline \multicolumn{1}{|c||}{} & \multicolumn{1}{c||}{$p_{\text {Lab }}$} & \multicolumn{3}{c||}{} & \multicolumn{4}{c||}{ Projectile fragments } & \multicolumn{4}{c|}{ Target fragments } \\
$(\mathrm{A} \mathrm{TeV} / c)$ & $\left\langle N_{s}\right\rangle$ & $\left\langle N_{g}\right\rangle$ & $\left\langle N_{b}\right\rangle$ & $\left\langle N_{p}\right\rangle$ & $\left\langle N_{n}\right\rangle$ & $\left\langle N_{\gamma}\right\rangle$ & $\left\langle N_{h f}\right\rangle$ & $\left\langle N_{p}\right\rangle$ & $\left\langle N_{n}\right\rangle$ & $\left\langle N_{\gamma}\right\rangle$ & $\left\langle N_{h f}\right\rangle$ \\
\hline \hline 0.2 & 45.4 & 1.3 & 2.1 & 0.74 & 0.80 & 0.20 & 1.8 & 0.64 & 0.70 & 0.13 & 1.5 \\
\hline & 43.9 & 1.4 & 2.0 & 0.73 & 0.82 & 0.19 & 1.7 & 0.62 & 0.68 & 0.12 & 1.4 \\
\hline 20 & 144.8 & 1.3 & 1.9 & 0.85 & 0.90 & 0.17 & 1.7 & 0.68 & 0.71 & 0.11 & 1.5 \\
\hline & 126.0 & 1.4 & 2.0 & 0.80 & 0.93 & 0.16 & 1.8 & 0.65 & 0.76 & 0.11 & 1.4 \\
\hline 2000 & 360.6 & 1.2 & 1.9 & 0.91 & 0.95 & 0.14 & 1.7 & 0.71 & 0.80 & 0.10 & 1.4 \\
\hline 200000 & 751.6 & 1.2 & 1.9 & 0.91 & 0.98 & 0.12 & 1.6 & 0.72 & 0.79 & 0.09 & 1.4 \\
\hline
\end{tabular}




\section{Figure Captions}

1. Photon spectrum resulting from the reaction $\operatorname{Ti}(\mathrm{n}, \mathrm{x})$ at $19 \mathrm{MeV}$. The dashed histogram represents PEANUT results with errors. Dots are experimental data from [34].

2. As in Fig.1 for Tungsten. Experimental points are from [35].

3. Grey particle multiplicity distributions for interactions of oxygen (a) and sulfur (b) with emulsion nuclei are plotted together with experimental results of the EMU01Collab. 24, 36] and of the KLM-Collab. 25].

4. Grey particle multiplicity distributions in gold-emulsion interactions are shown in comparison with data of the EMU01-Collab. 222.

5. Black particle multiplicity distributions for interactions of oxygen (a) and sulfur (b) with emulsion nuclei are plotted together with experimental results of the EMU01Collab. 24, 36] and of the KLM-Collab. [25].

6. Black particle multiplicity distributions in gold-emulsion interactions are shown in comparison with data of the EMU01-Collab. 24].

7. Correlations between grey $\left(N_{g}\right)$, black $\left(N_{b}\right)$, and shower $\left(N_{s}\right)$ particle multiplicities in interactions of sulfur with emulsion nuclei are compared to experimental results [24].

8. The correlations between grey $\left(N_{g}\right)$ and black $\left(N_{b}\right)$ particle multiplicities in sulfuremulsion interactions are compared to experimental results [24.

9. Angular distributions of grey $(a, b)$ and black (c,d) particles in oxygen- and sulfuremulsion interactions are shown together with data [21, 25].

10. Multiplicity distributions of slow protons and singly charged fragments from oxygennucleus interactions are compared to results of the WA80-Collab. [26]. See the text for kinematical cuts applied.

11. Mass distributions of residual target nuclei produced in carbon-silver interactions at 25.2 GeV/nucleon as obtained with the model are compared to experimental results 29.

12. Cross sections for the production of residual projectile nuclei in interactions of oxygen (a) and iron (b) nuclei with air (nitrogen) are shown for two different laboratory energies.

13. Pseudorapidity distributions of photons produced in oxygen- (a) and iron-air (b) interactions at laboratory energies of $0.2 \mathrm{TeV} /$ nucleon (filled symbols) and of $2000 \mathrm{TeV} /$ nucleon (open symbols) are given. We show the contributions of photons resulting from decays and those from deexcitation processes of the projectile prefragments separately. The distributions are plotted in the nucleon-nucleon c.m. frame. 
14. Feynman- $x_{F}$-distributions of photons produced in iron-air interactions at $200000 \mathrm{TeV} / \mathrm{nucleon}$ are shown. In (a) the contributions of photons from decays (stars) and from deexcitation processes of the projectile prefragments (line) as calculated within our model are presented. In (b) only deexcitation photons are plotted.

15. Cross sections for the production of residual nuclei in lead-lead collisions at an energy of $200 \mathrm{GeV}$ in the nucleon-nucleon c.m. system. Shown is the fragmentation yield for one of the colliding nuclei. 


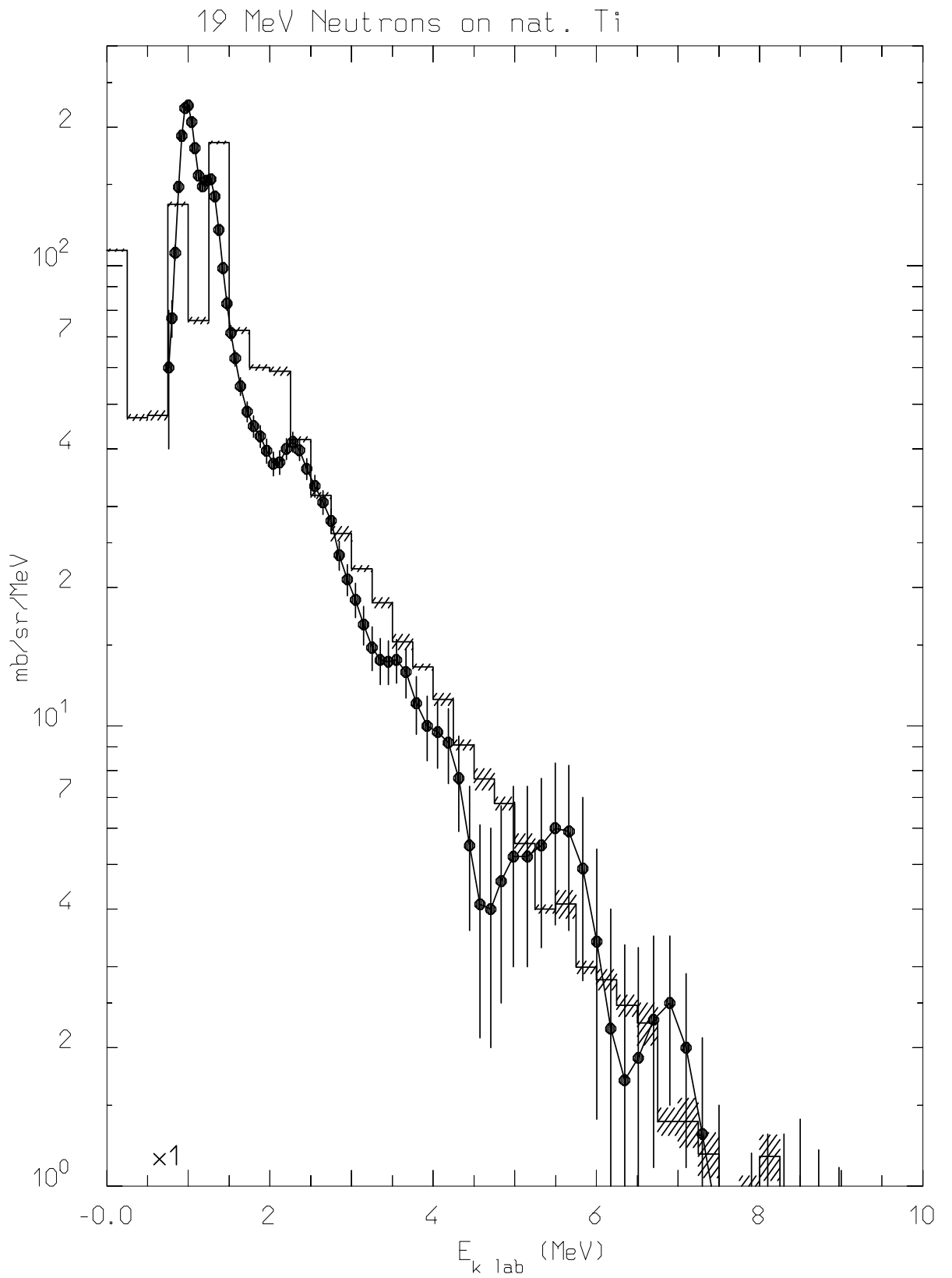

Fig. 1 


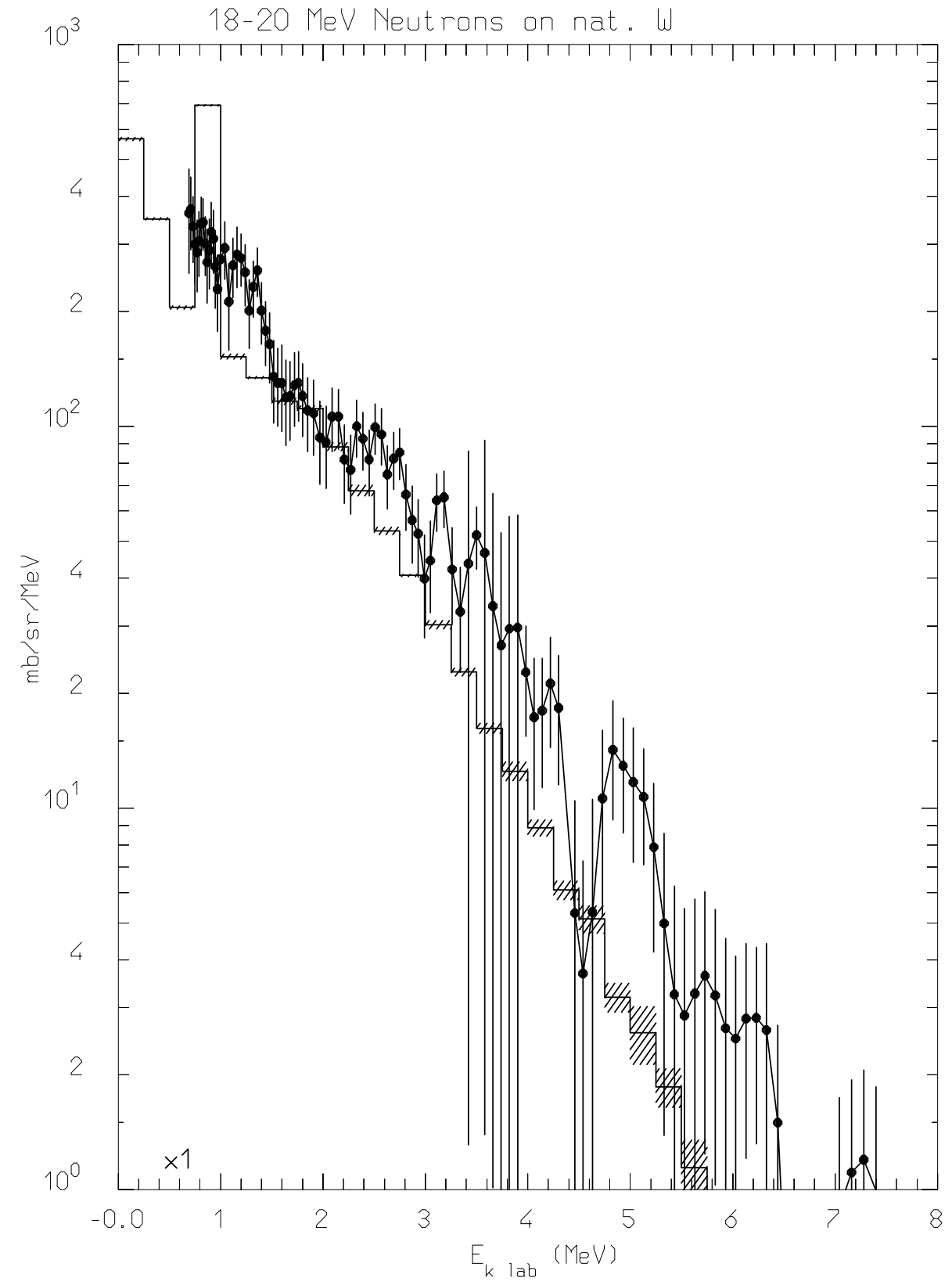

Fig. 2 


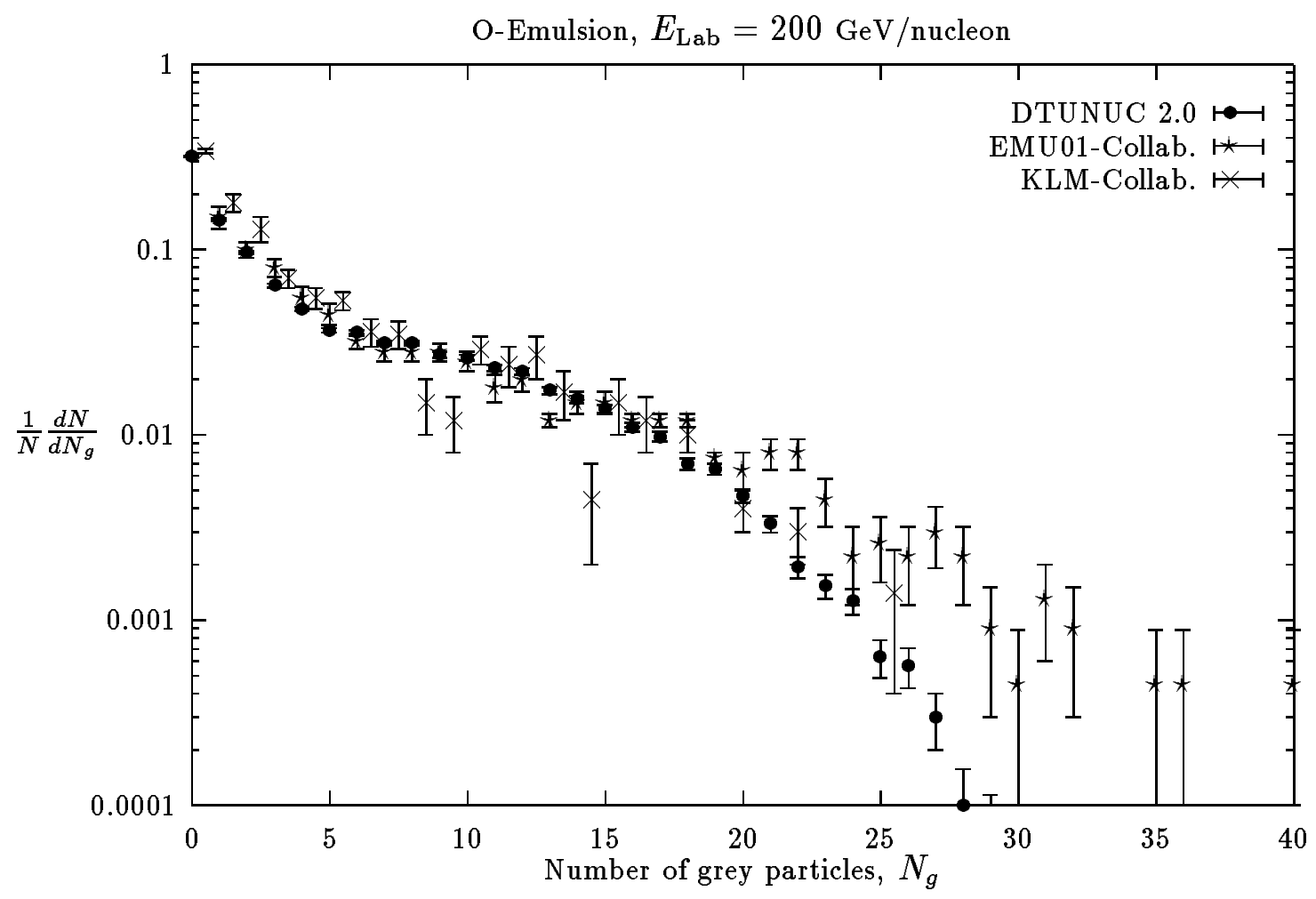

a)

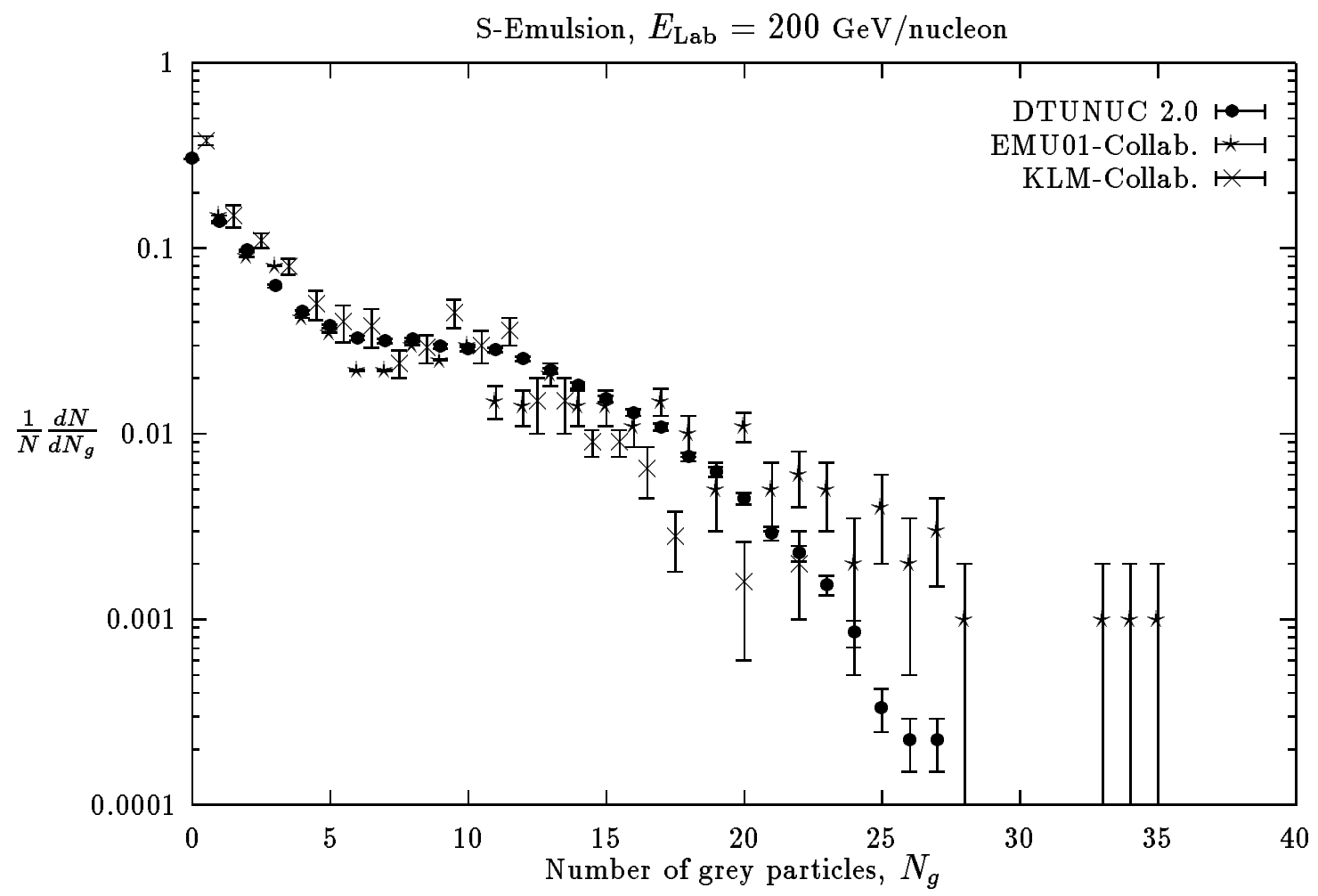

b)

Fig. 3 


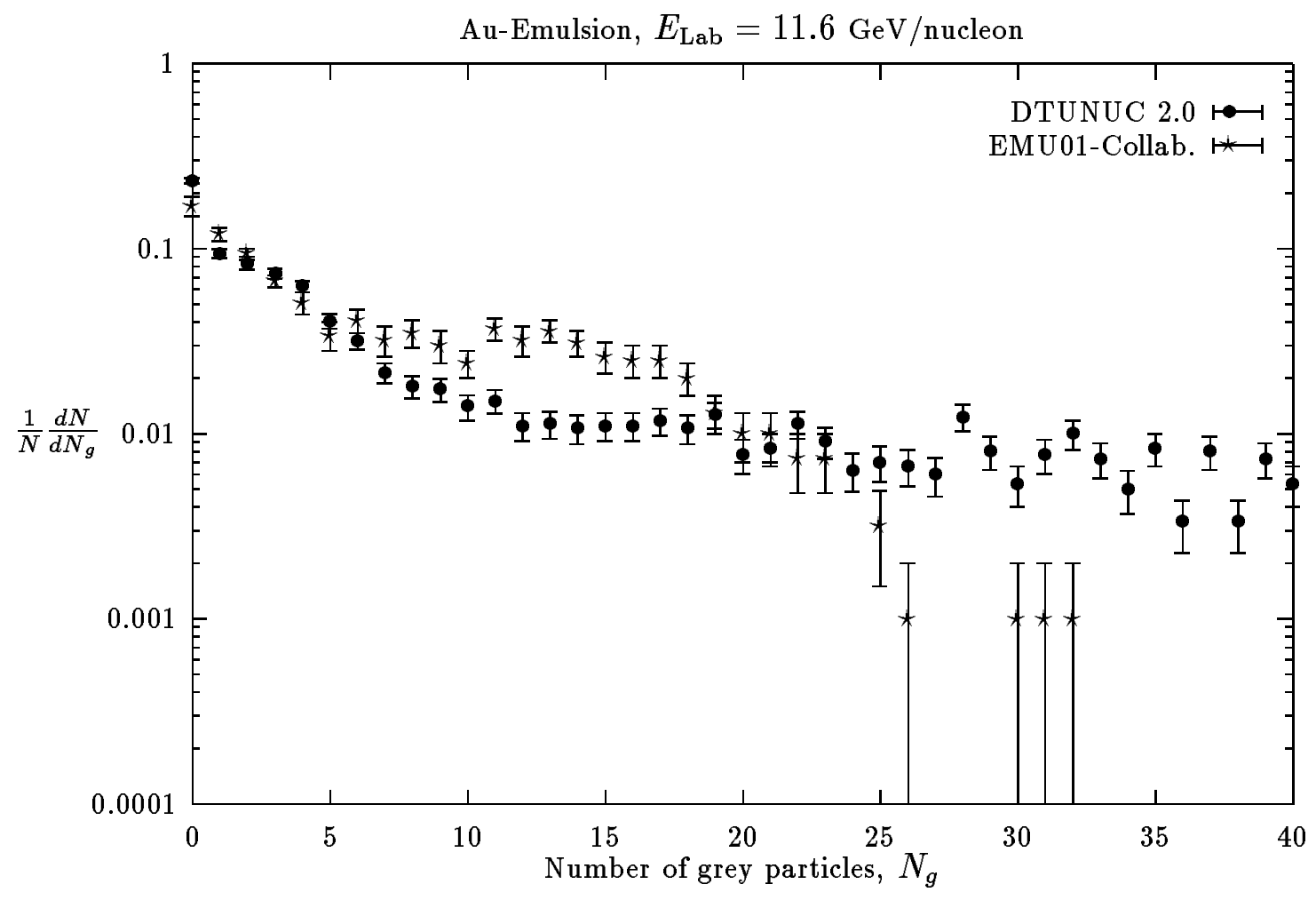

Fig. 4 


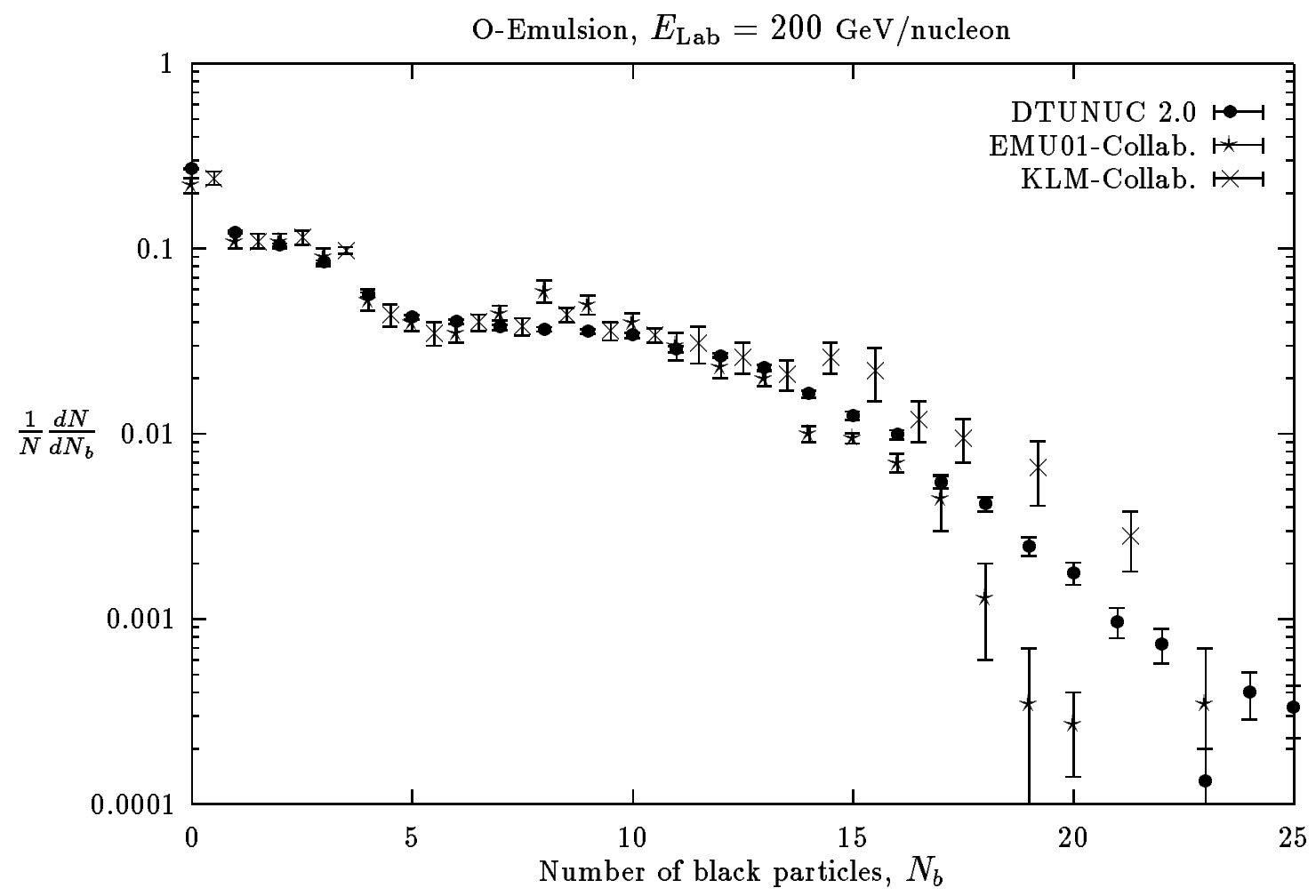

a)

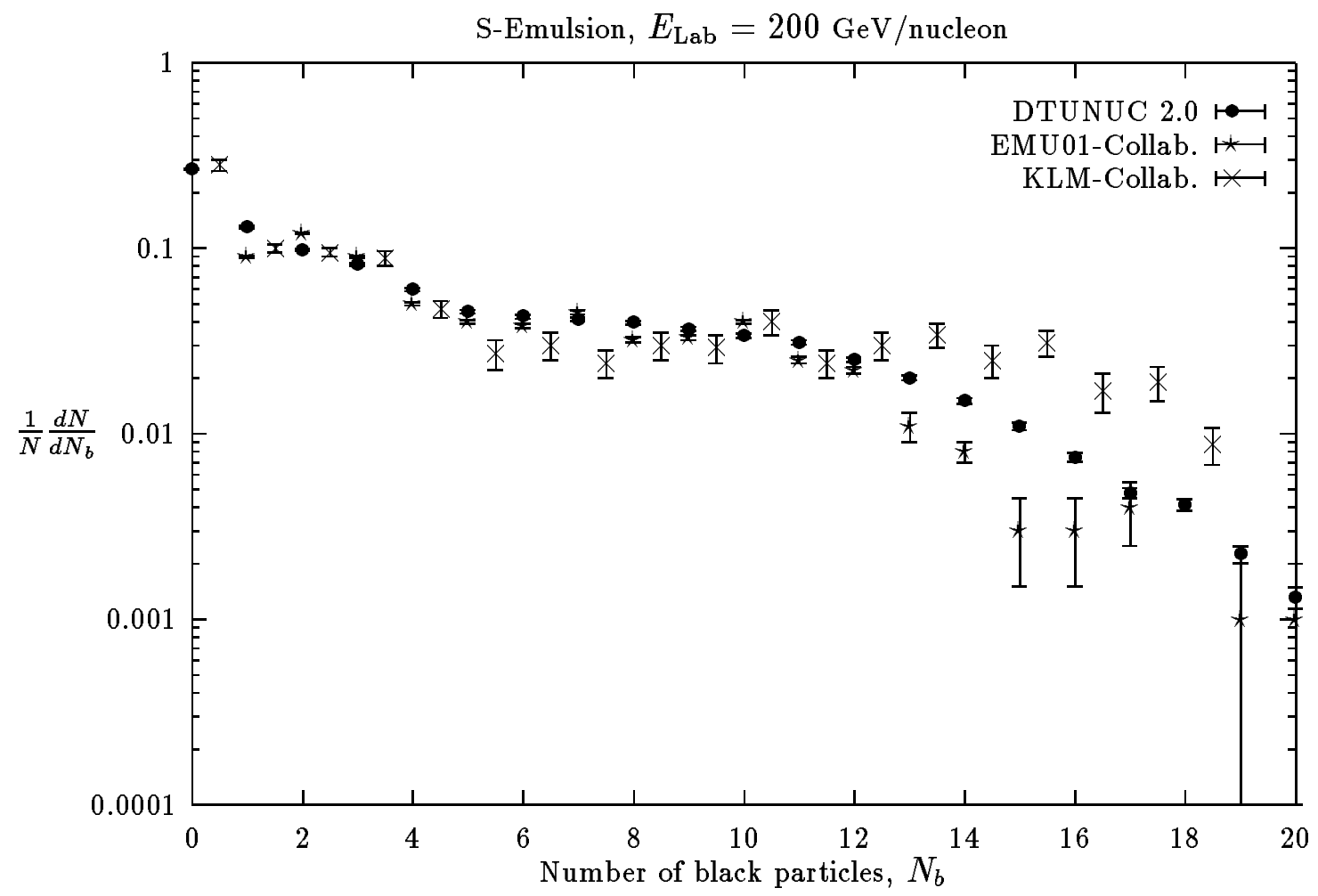

b)

Fig. 5 


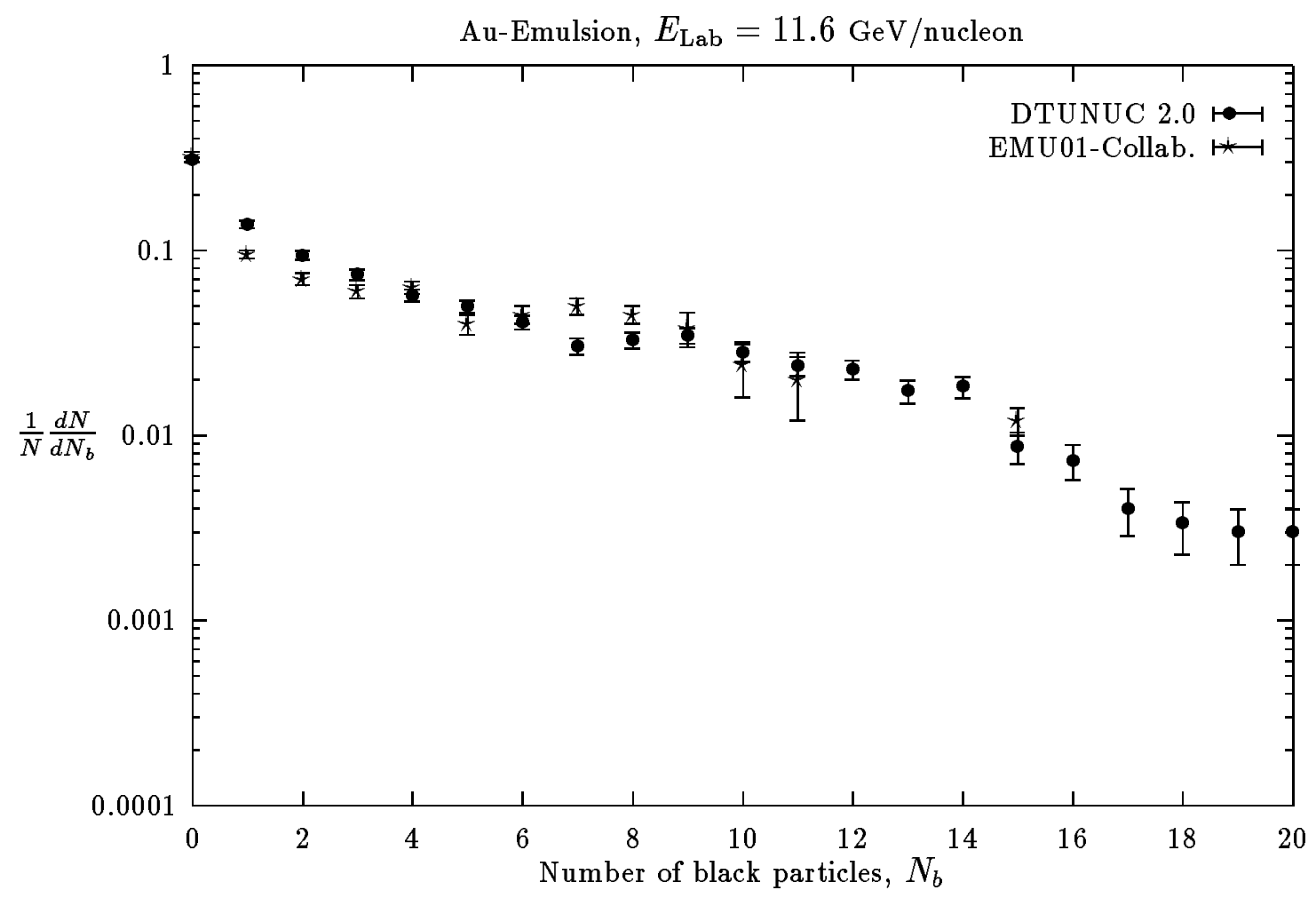

Fig. 6 


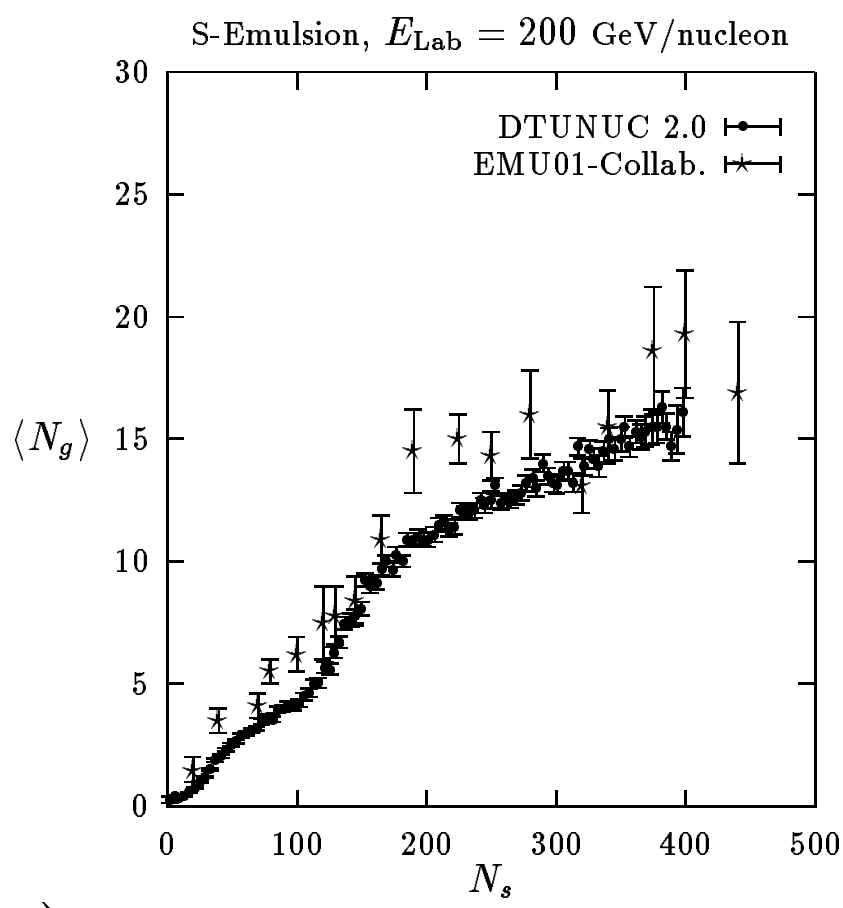

a)

b)
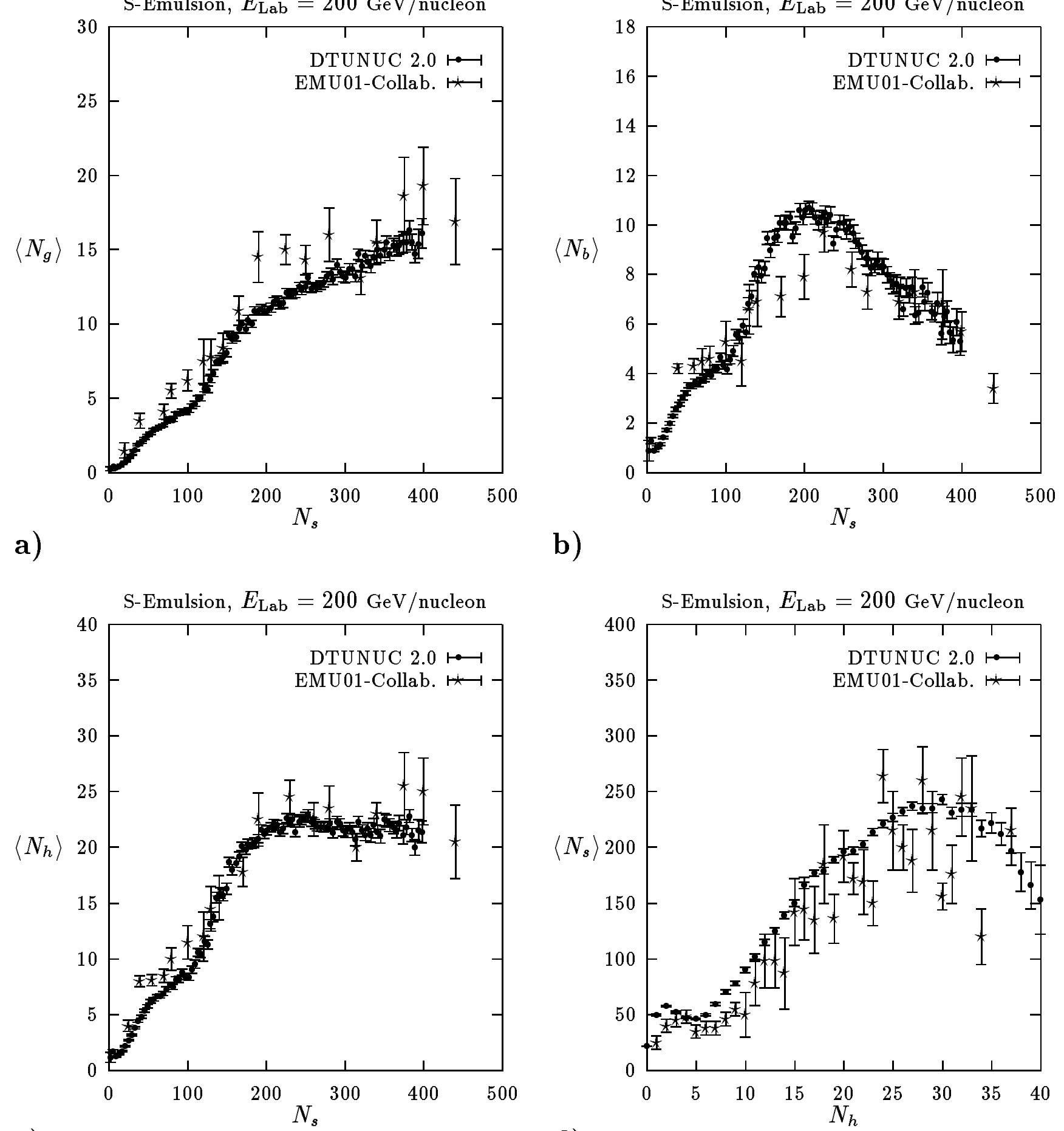

c)

d)

Fig. 7 

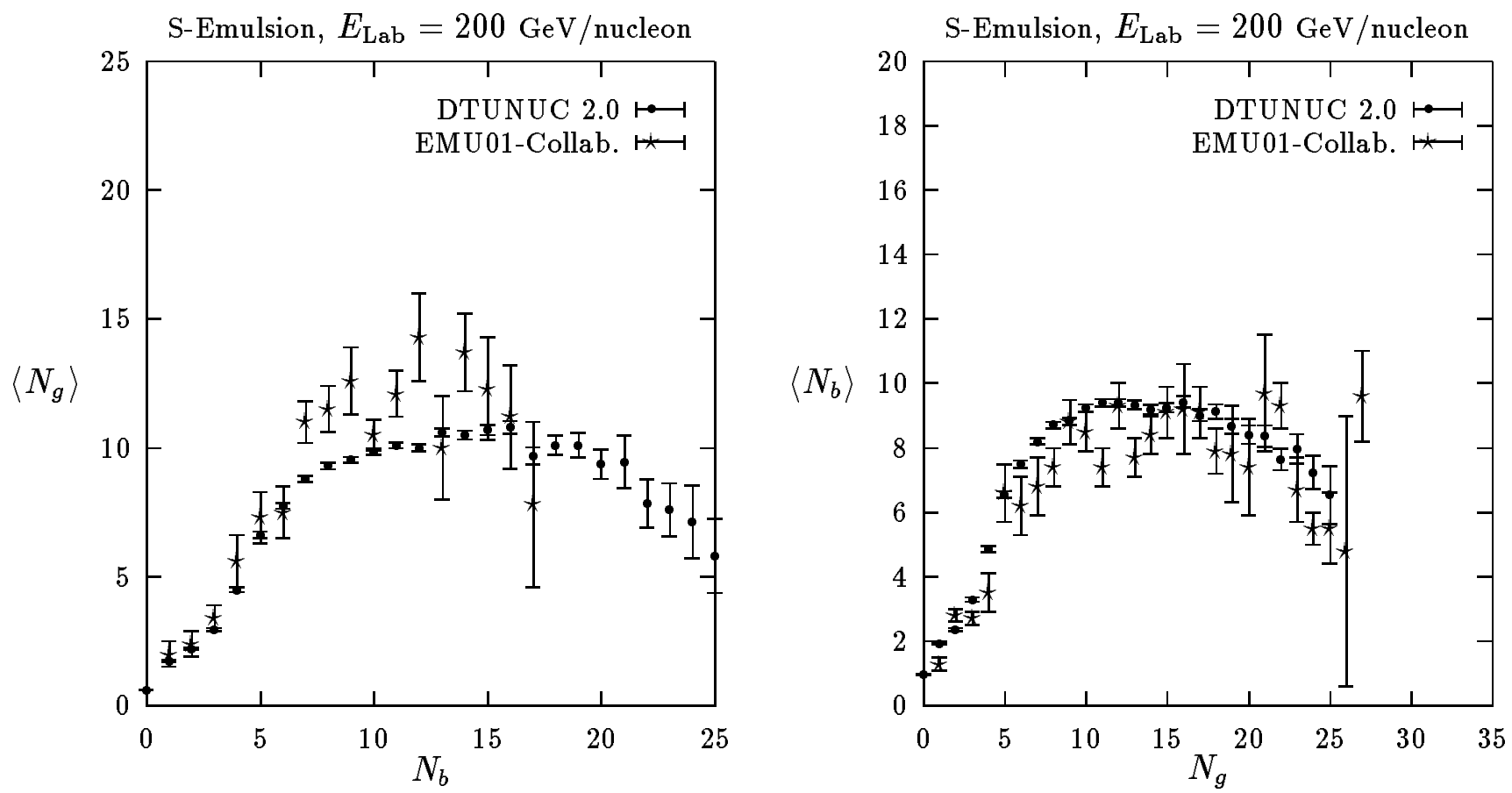

a)

b)

Fig. 8 


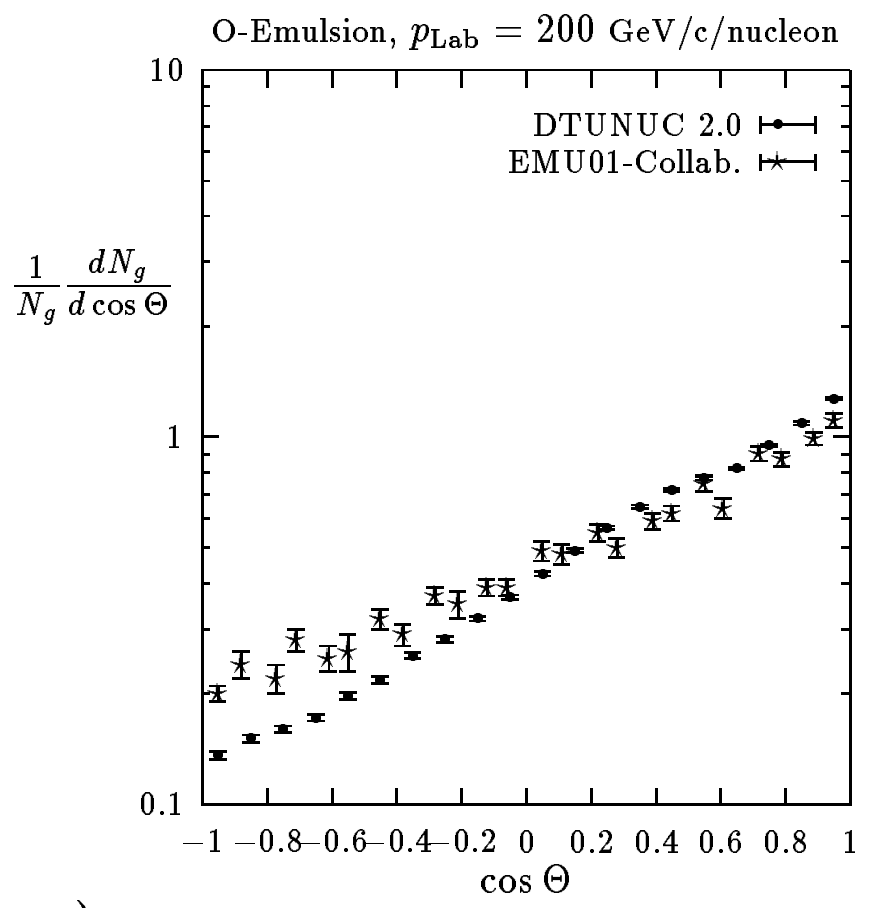

a)

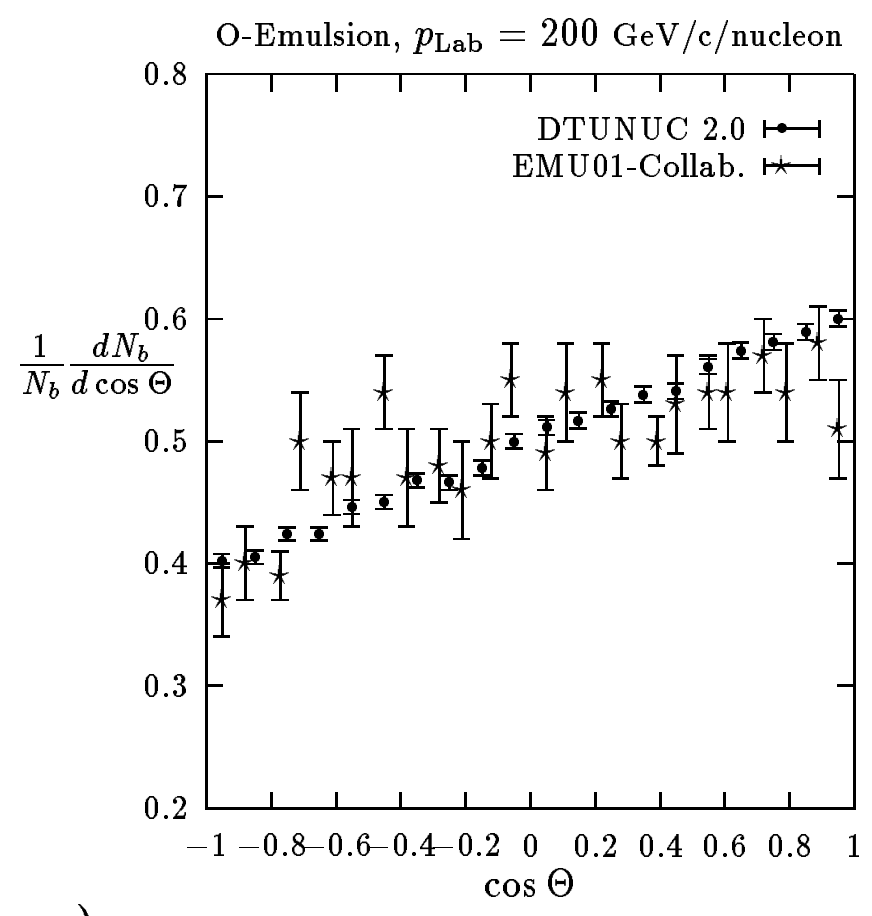

S-Emulsion, $p_{\text {Lab }}=200 \mathrm{GeV} / \mathrm{c} /$ nucleon

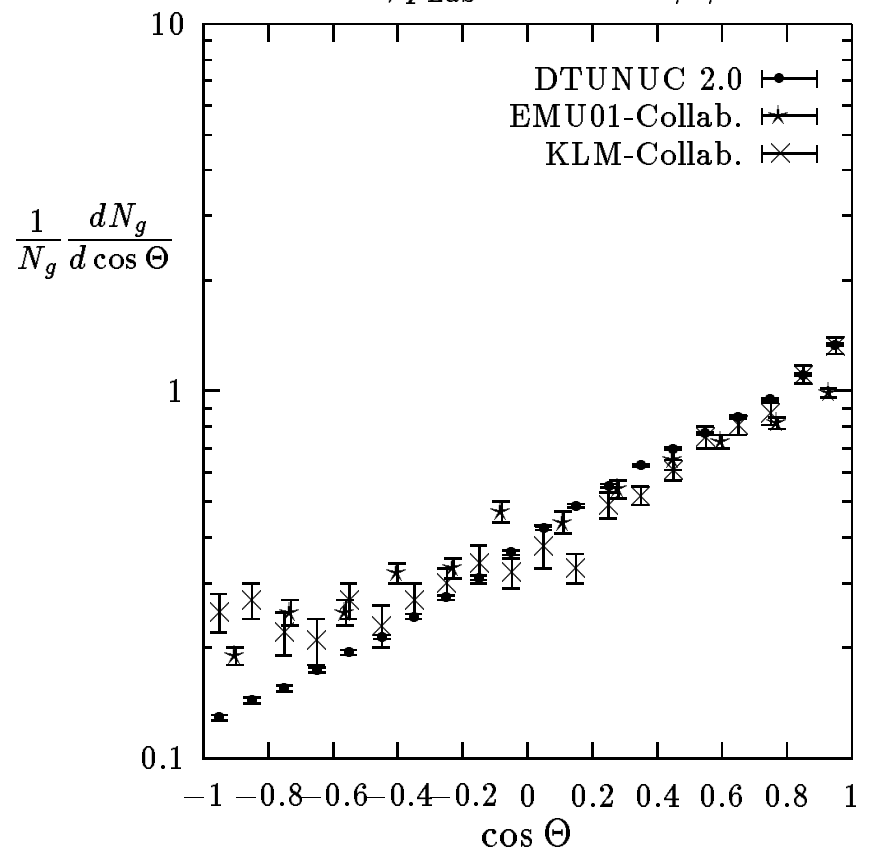

b)

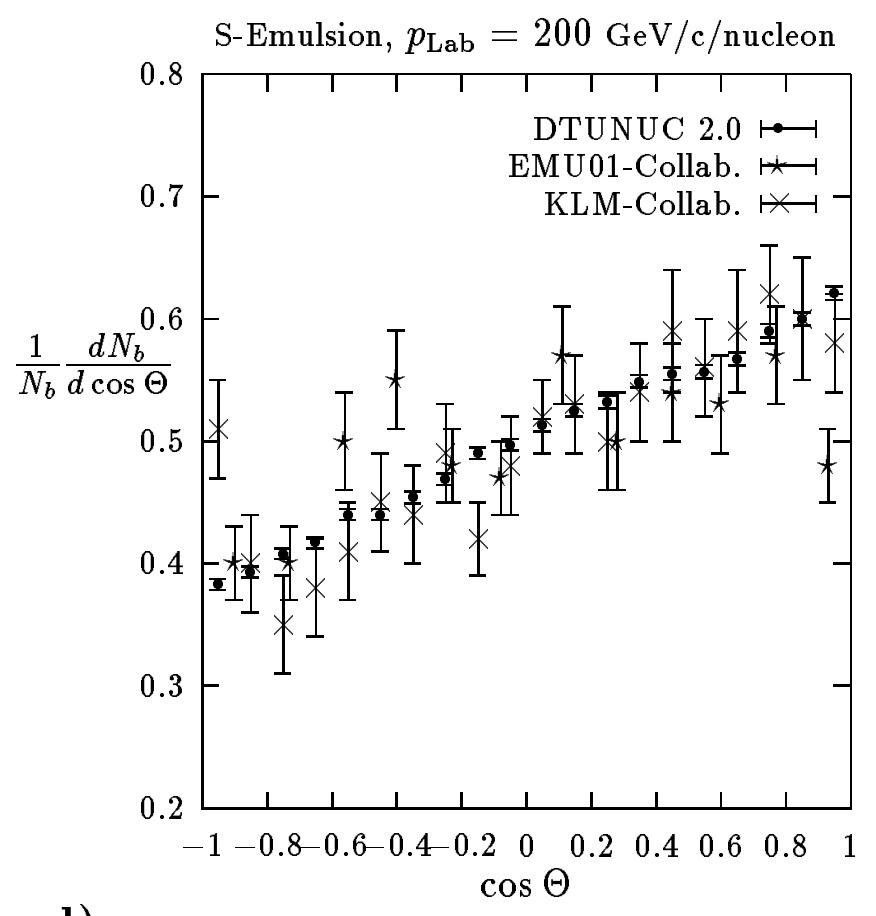

Fig. 9 


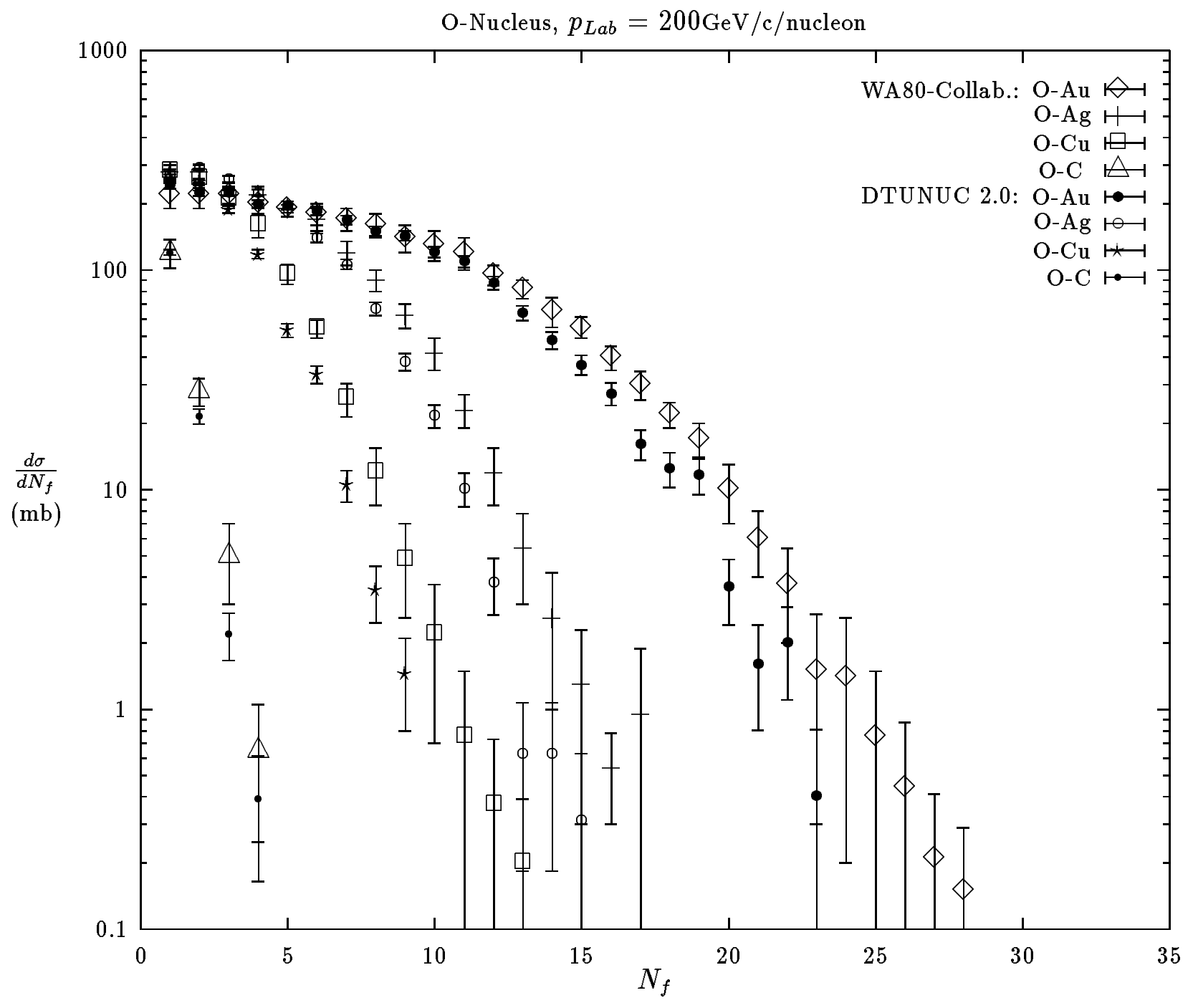

Fig. 10 


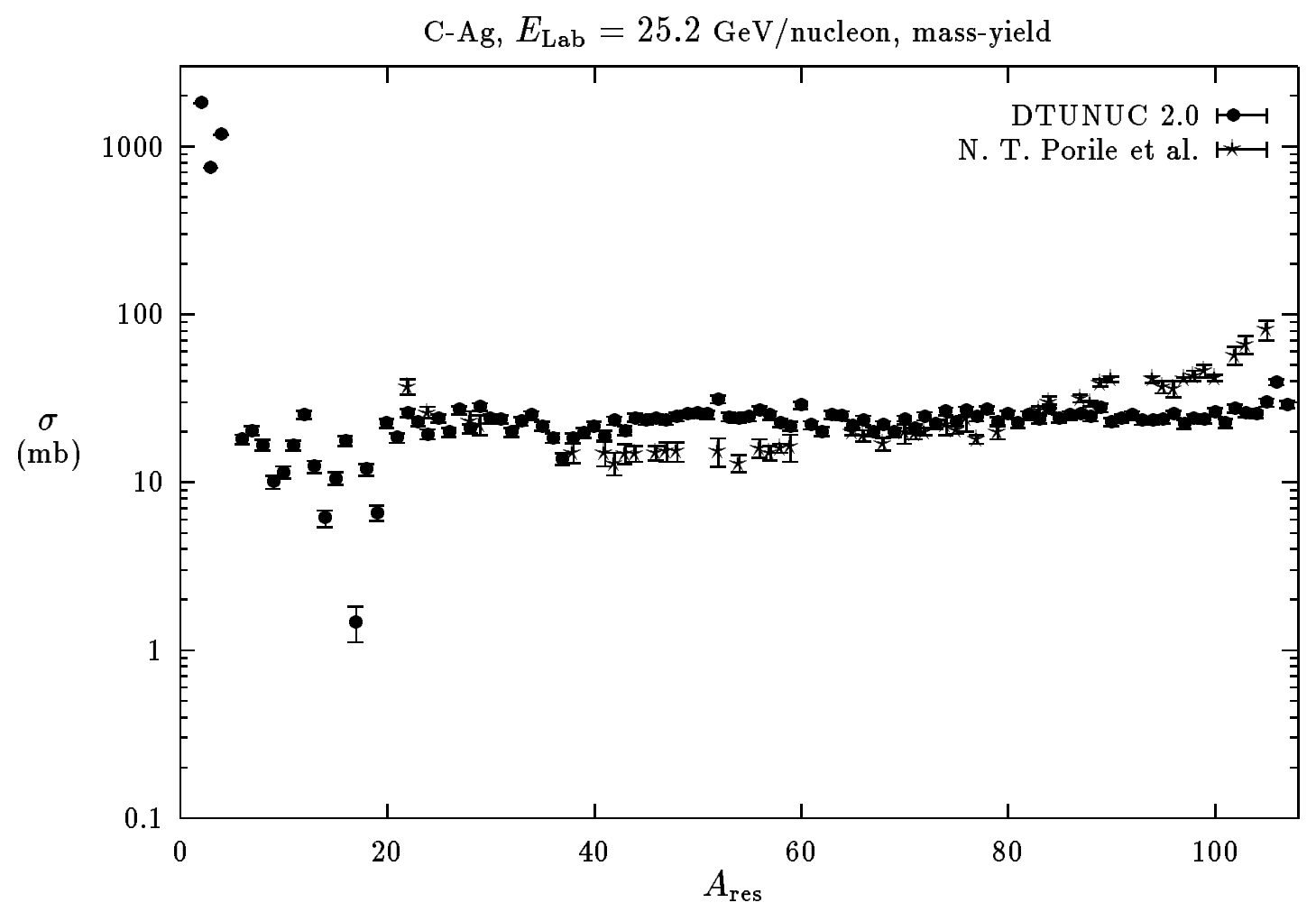

Fig. 11 
O-Air, mass-yield of residual O-nuclei

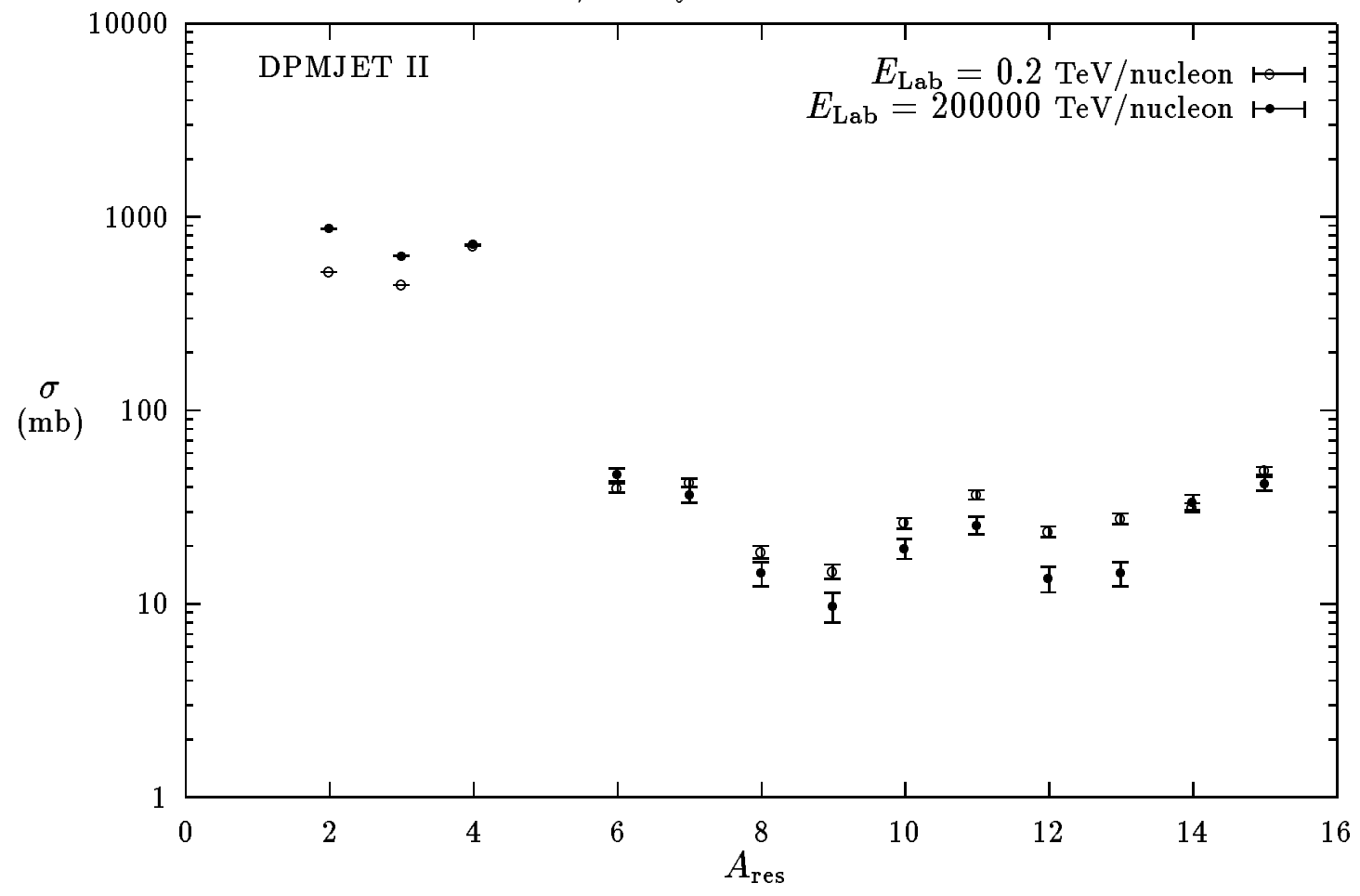

a)

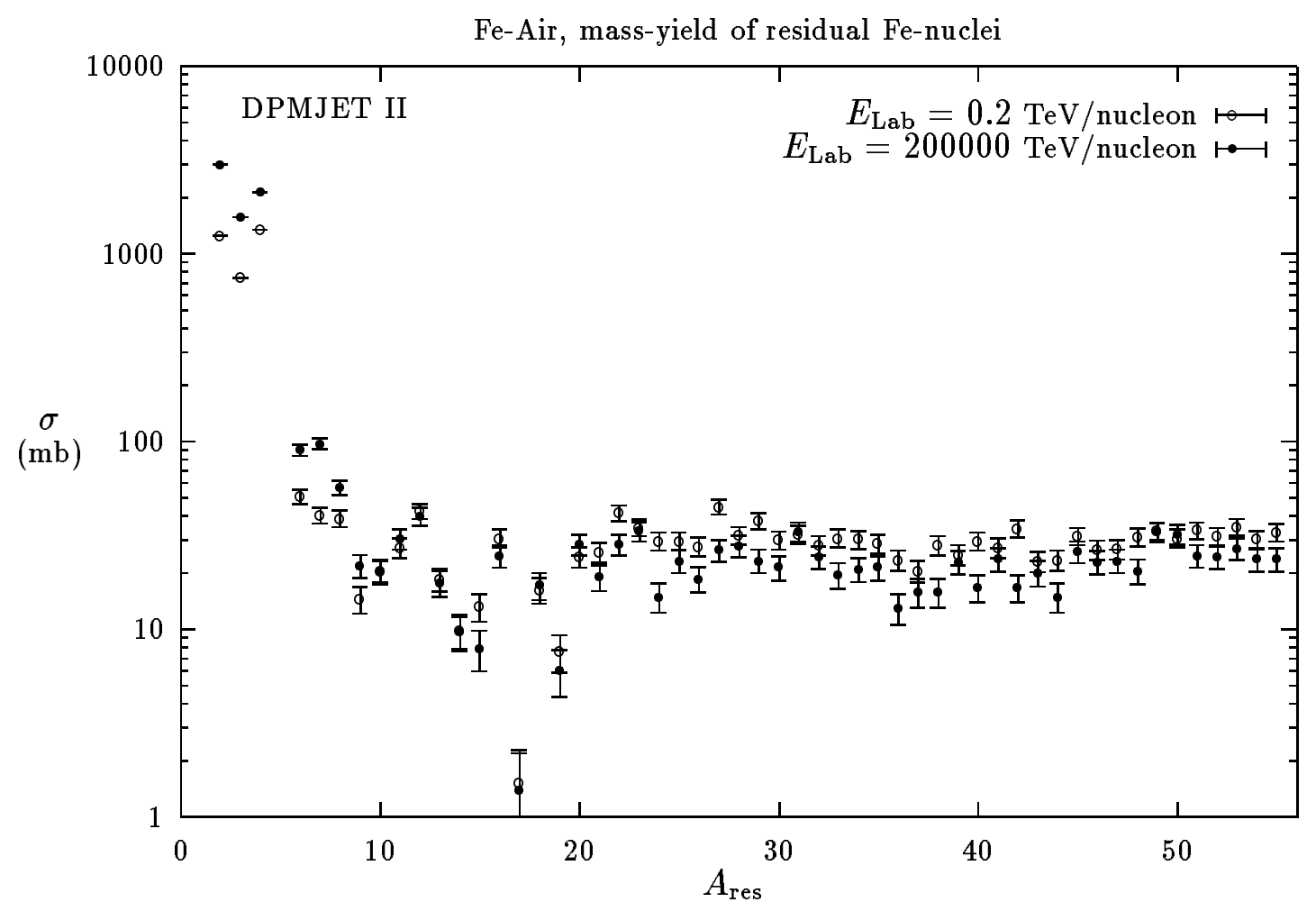

b)

Fig. 12 


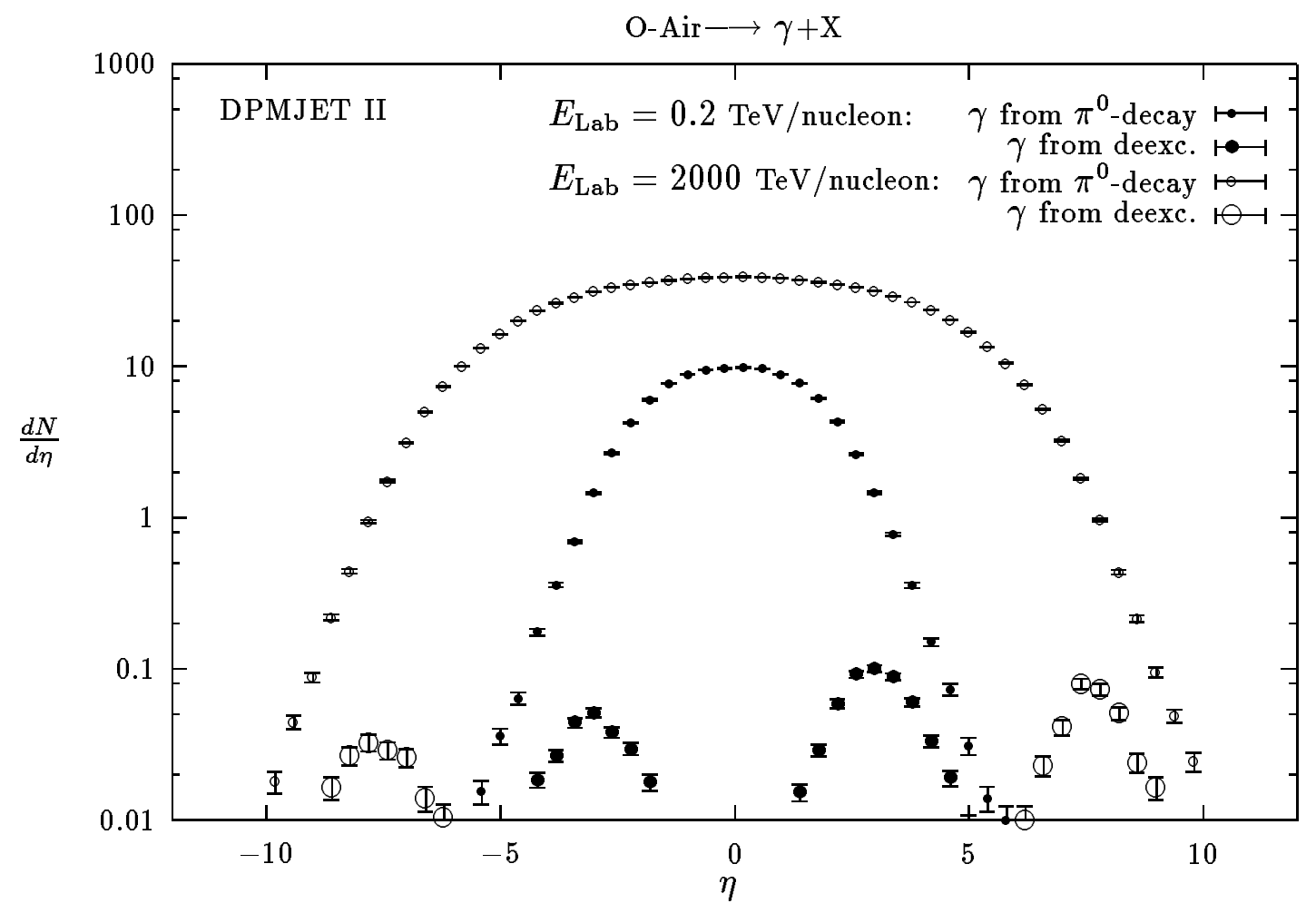

a)

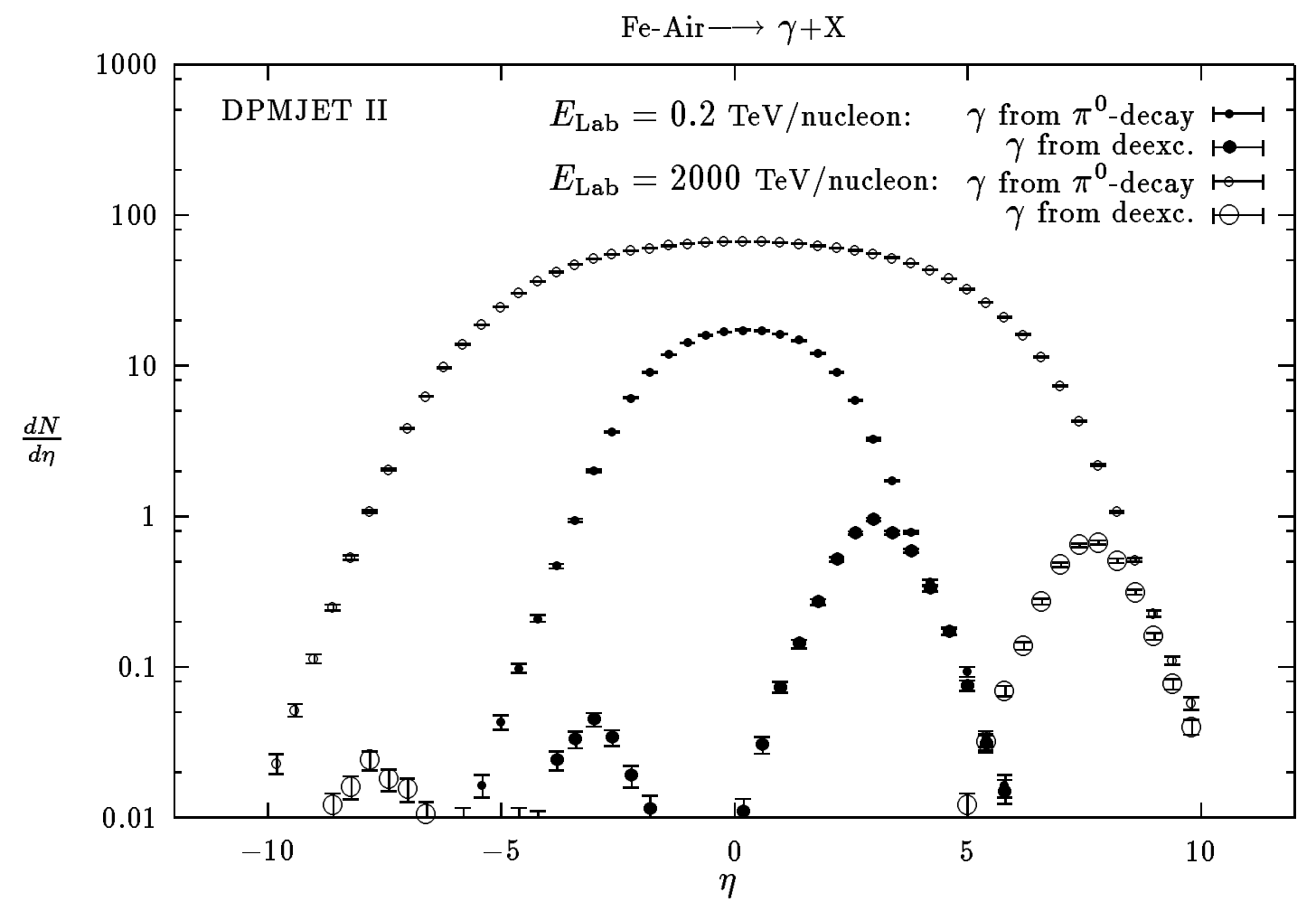

b)

Fig. 13 


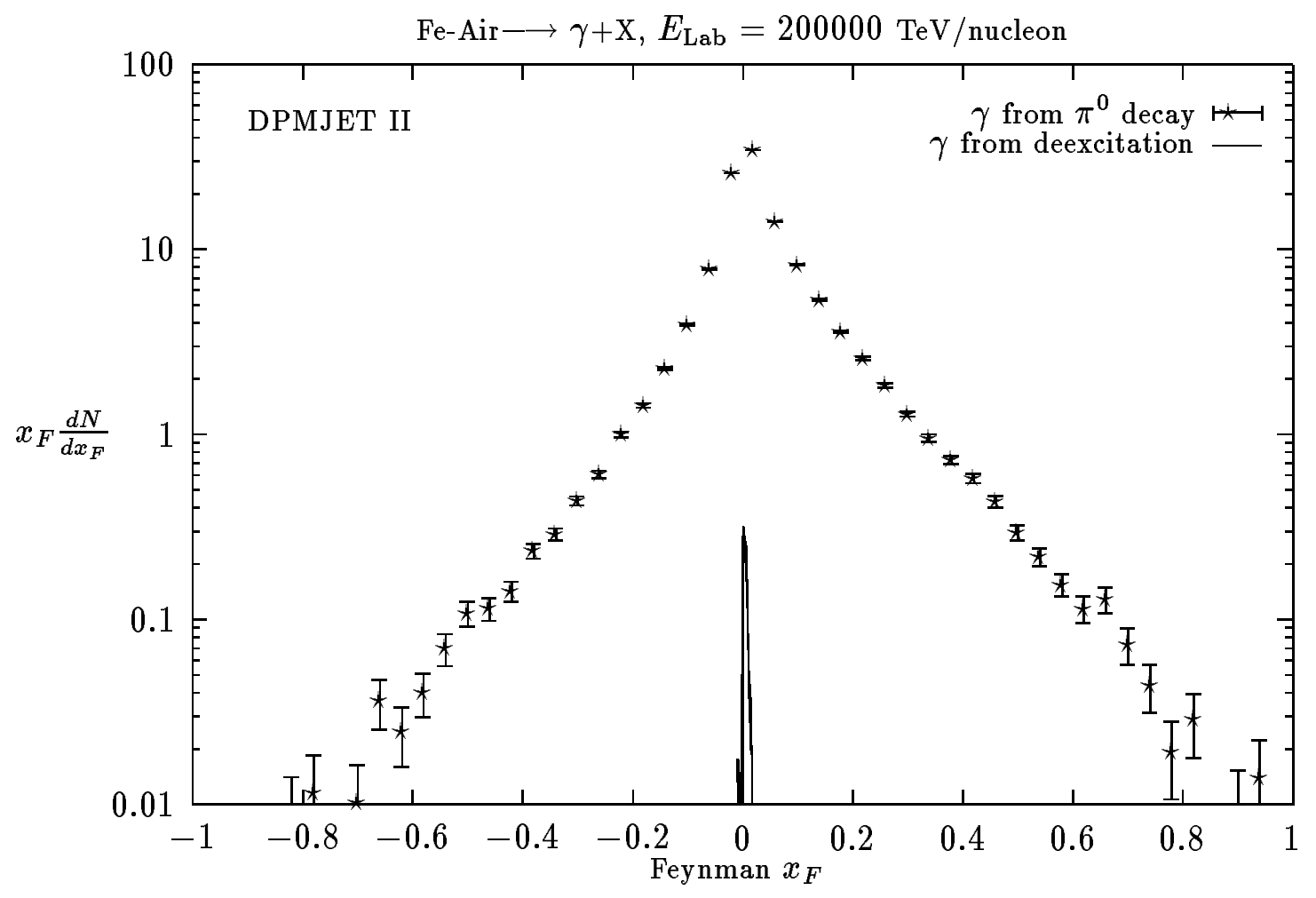

a)

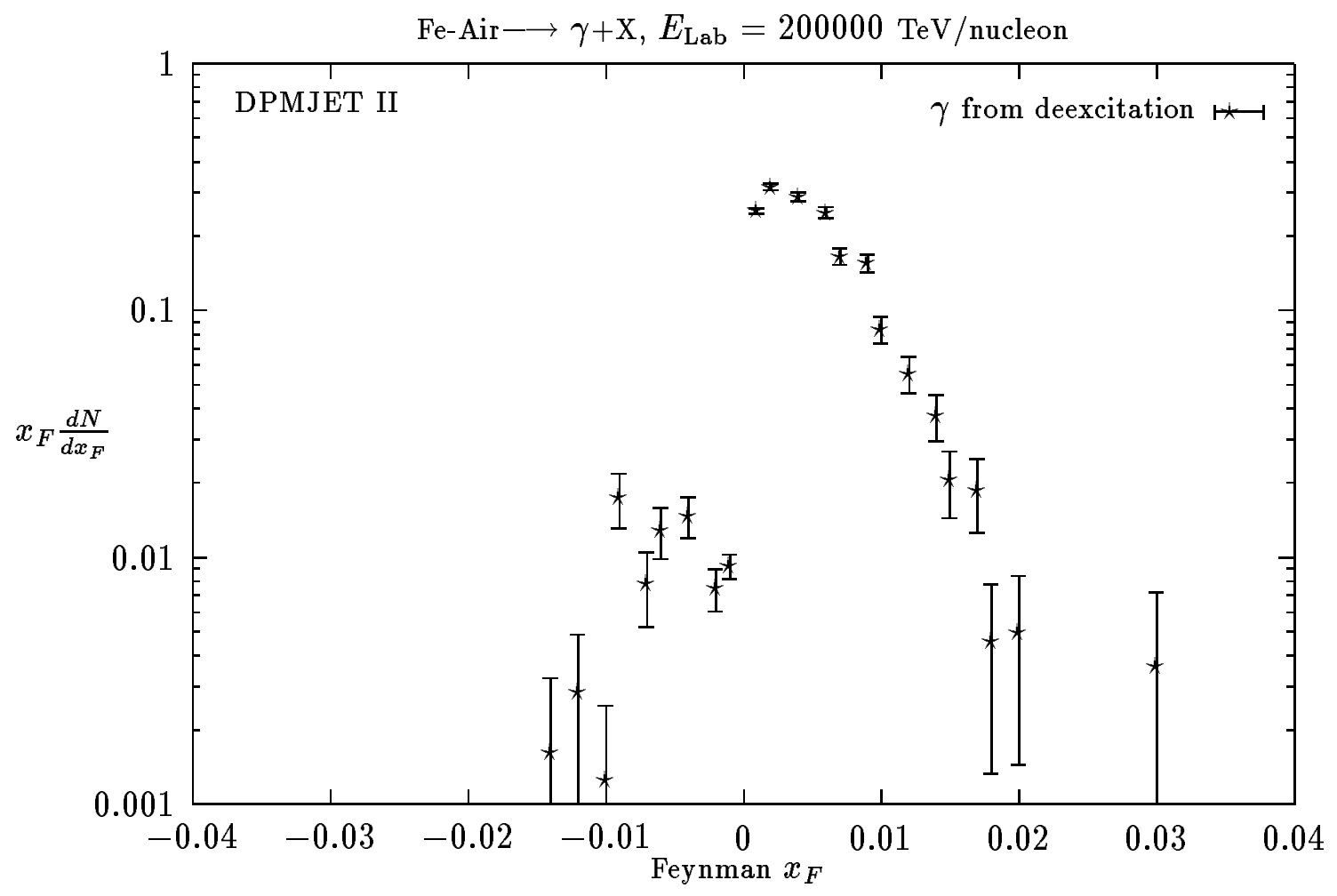

b)

Fig. 14 


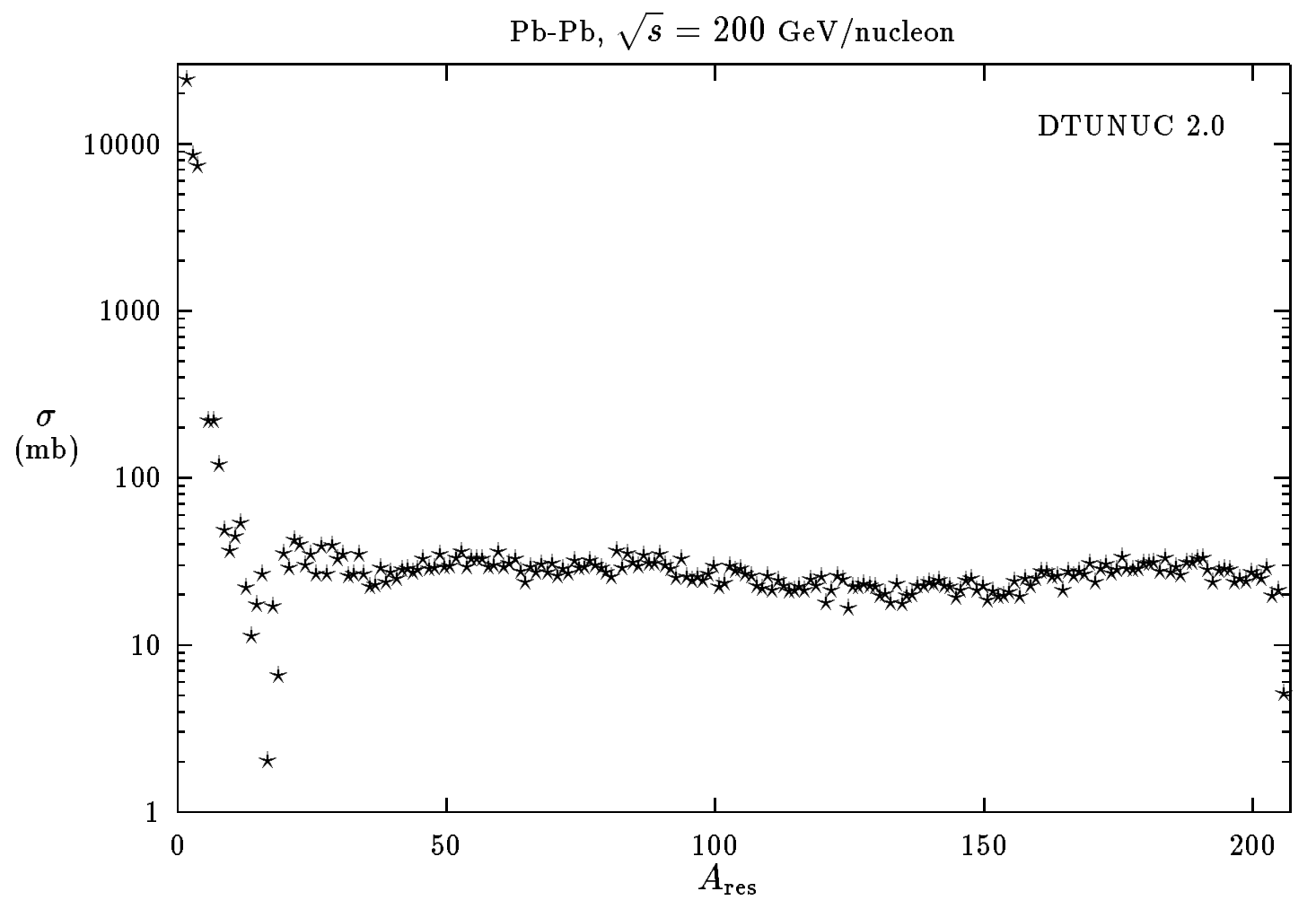

Fig. 15 